\title{
Note on the existence theory for pseudo-monotone evolution problems
}

\author{
A. KALtenbaCH AND M. RƯŽIČKA
}

Abstract. In this note, we develop a framework which allows to prove an existence result for nonlinear evolution problems involving time-dependent, pseudo-monotone operators. This abstract existence result is applicable to a large class of concrete problems where the standard theorem on evolutionary pseudomonotone operators (cf. Theorem 1.3) is not applicable. To this end we introduce the notion of Bochner pseudo-monotonicity, and Bochner coercivity, which are appropriate extensions of the concepts of pseudomonotonicity and coercivity to the evolutionary setting. Moreover, we give sufficient conditions for these new notions, which are easily and widely applicable.

\section{Introduction}

The theory of pseudo-monotone operators turned out to be a powerful instrument in proving existence results for nonlinear problems both in the time-independent and the time-dependent setting. The celebrated main theorem on pseudo-monotone operators, stemming from Brezis [4], states the following ${ }^{1}$

Theorem 1.1. Given a reflexive, separable Banach space $V$, a right-hand side $f \in V^{*}$ and an operator $A: V \rightarrow V^{*}$ that it is pseudo-monotone, bounded and coercive, there exists a solution $u \in V$ of the problem

$$
A u=f \quad \text { in } V^{*},
$$

i.e., the operator A is surjective.

Typical examples for such operators are sums of a monotone and a compact operator (cf. [24]). Thus, the theory of pseudo-monotone operators extends the theory of monotone operators due to Browder [5] and Minty [15].

There exists also a time-dependent analogue of the above result (cf. [21]).

Theorem 1.3. Given an evolution triple $V \hookrightarrow H \cong H^{*} \hookrightarrow V^{*}$, a finite time horizon $I:=(0, T)$, an initial value $\boldsymbol{y}_{0} \in H$, a right-hand side $\boldsymbol{f} \in\left(L^{p}(I, V)\right)^{*}, 1<p<\infty$,

Mathematics Subject Classification: 47 H05, 35 K90, 35 A01

Keywords: Evolution equation, Pseudo-monotone operator, Existence result.

${ }^{1}$ All notion are defined in Sect. 2. 
and a family of operators $A(t): V \rightarrow V^{*}, t \in I$, such that the induced operator $\mathcal{A}: L^{p}(I, V) \rightarrow\left(L^{p}(I, V)\right)^{*}$, given via

$$
\langle\mathcal{A} \boldsymbol{v}, \boldsymbol{w}\rangle_{L^{p}(I, V)}:=\int_{I}\langle A(t)(\boldsymbol{v}(t)), \boldsymbol{w}(t)\rangle_{V} d t
$$

for every $\boldsymbol{v}, \boldsymbol{w} \in L^{p}(I, V)$, is pseudo-monotone, bounded and coercive, there exists a solution $\boldsymbol{y} \in W:=\left\{\boldsymbol{u} \in L^{p}(I, V) \mid \boldsymbol{u}^{\prime} \in\left(L^{p}(I, V)\right)^{*}\right\}$ of the initial value problem

$$
\begin{aligned}
\boldsymbol{y}^{\prime}+\mathcal{A} \boldsymbol{y} & =\boldsymbol{f} & & \text { in }\left(L^{p}(I, V)\right)^{*}, \\
\boldsymbol{y}(0) & =\boldsymbol{y}_{0} & & \text { in } H .
\end{aligned}
$$

This result is essentially a consequence of the main theorem on pseudo-monotone perturbations of maximal monotone mappings, stemming from Browder [6] and Brezis [4] (see also [24, §32.4.]). In doing so, one interprets the time derivative as a maximal monotone mapping

$$
\begin{aligned}
& L: D(L) \subseteq L^{p}(I, V) \rightarrow\left(L^{p}(I, V)\right)^{*} \text { with } L \boldsymbol{x}:=\boldsymbol{x}^{\prime} \\
& \text { and } D(L):=\{\boldsymbol{x} \in W \mid \boldsymbol{x}(0)=\mathbf{0} \text { in } H\} .
\end{aligned}
$$

Note that the maximal monotonicity of the operator $L$ can basically be traced back to the generalized integration by parts formula (cf. Proposition 2.23). For details we refer to $[8,14,18,19,21,24]$.

To illustrate the applicability of the above existence results we consider as prototypical applications the steady and unsteady motion of incompressible shear dependent fluids in a bounded domain $\Omega \subset \mathbb{R}^{3}$. The motion in the steady and unsteady situation, resp., is governed by

$$
\begin{array}{rlrl}
-\operatorname{div} \mathbf{S}(\mathbf{D u})+[\nabla \mathbf{u}] \mathbf{u}+\nabla \pi & =\mathbf{f} & & \text { in } \Omega, \\
\operatorname{div} \mathbf{u}=0 & & \text { in } \Omega,
\end{array}
$$

and

$$
\begin{aligned}
\partial_{t} \mathbf{u}-\operatorname{div} \mathbf{S}(\mathbf{D u})+[\nabla \mathbf{u}] \mathbf{u}+\nabla \pi & =\mathbf{f} & & \text { in } I \times \Omega, \\
\operatorname{div} \mathbf{u} & =0 & & \text { in } I \times \Omega, \\
\mathbf{u}(0) & =\mathbf{u}_{0} & & \text { in } \Omega,
\end{aligned}
$$

respectively. Here, $\mathbf{u}=\left(u_{1}, u_{2}, u_{3}\right)^{\top}$ is the fluid velocity, Du its symmetric gradient, i.e., $\mathbf{D u}=\frac{1}{2}\left(\nabla \mathbf{u}+\nabla \mathbf{u}^{\top}\right), \mathbf{S}(\mathbf{D u})=(\delta+|\mathbf{D u}|)^{p-2} \mathbf{D u}, p \in(1, \infty), \delta \geq 0$, the extra stress tensor, $\pi$ the pressure, $\mathbf{f}=\left(f_{1}, f_{2}, f_{3}\right)^{\top}$ the external body force, and $\mathbf{u}_{0}$ the initial velocity. Here, we used the notation $([\nabla \mathbf{u}] \mathbf{u})_{i}=\sum_{j=1}^{3} u_{j} \partial_{j} u_{i}, i=1,2,3$, for the convective term. We denote by $V:=W_{0, \operatorname{div}}^{1, p}(\Omega)$ and $H:=L_{\operatorname{div}}^{2}(\Omega)$ the spaces of divergence free vector fields, i.e., $W_{0, \operatorname{div}}^{1, p}(\Omega)$ is the closure of $\mathcal{V}=\left\{\mathbf{v} \in C_{0}^{\infty}(\Omega)^{3} \mid \operatorname{div} \mathbf{v}=0\right\}$ with respect to the gradient norm $\|\nabla \cdot\|_{L^{p}(\Omega)^{3 \times 3}}$ and $L_{\text {div }}^{2}(\Omega)$ the closure of $\mathcal{V}$ with respect to $\|\cdot\|_{L^{2}(\Omega)^{3}}$. Then, the first two terms in (1.6) define operators $S, B: V \rightarrow V^{*}$ 
through $\langle S \mathbf{u}, \mathbf{v}\rangle_{V}:=\int_{\Omega} \mathbf{S}(\mathbf{D u}) \cdot \mathbf{D v} \mathrm{d} x,\langle B \mathbf{u}, \mathbf{v}\rangle_{V}:=-\int_{\Omega} \mathbf{u} \otimes \mathbf{u} \cdot \mathbf{D v} \mathrm{d} x .^{2}$ Note that $\int_{\Omega} \nabla \pi \cdot \mathbf{v} \mathrm{d} x=0$ for $\mathbf{v} \in V$ and sufficiently smooth $\pi$. It is well known that the operator $S$ is strictly monotone, bounded, coercive and continuous (cf. [8,14]), and that, due to the compact embedding $W_{0}^{1, p}(\Omega) \hookrightarrow L^{q}(\Omega), q<\frac{3 p}{3-p}$, the operator $B$ is compact, bounded and $\langle B \mathbf{u}, \mathbf{u}\rangle_{V}=0$ if $p>9 / 5$ (cf. [14]). Thus, $A:=S+B$ fulfills the assumptions of Theorem 1.1, yielding the existence of solutions $\mathbf{u} \in V$ of (1.6) if $p>9 / 5$. To treat the unsteady problem (1.7) note that the operators $S, B$ induce operators $\mathcal{S}, \mathcal{B}: L^{p}(I, V) \rightarrow\left(L^{p}(I, V)\right)^{*}$ through (1.4). The induced operator $\mathcal{S}$ inherits the properties of the operator $S$ (cf. [24, Chapter 30]), i.e., $\mathcal{S}$ is a strictly monotone, bounded, coercive and continuous operator. On the other hand, the operator $\mathcal{B}$ is still bounded for sufficiently large $p$, e.g., $p>3$. However, the operator $\mathcal{B}$ is for no $p$ compact, ${ }^{3}$ since the embedding $L^{p}\left(I, W_{0}^{1, p}(\Omega)\right) \hookrightarrow L^{p}\left(I, L^{q}(\Omega)\right)$, $q<\frac{3 p}{3-p}$, is not compact. In fact, given $f_{n} \rightarrow f$ in $L^{p}(I)$ containing no strongly convergent subsequence and $\mathbf{g}_{n} \rightarrow \mathbf{g}$ in $W_{0}^{1, p}(\Omega)$, the sequence $\mathbf{u}_{n}(t):=f_{n}(t) \mathbf{g}_{n}$ is bounded in $L^{p}\left(I, W_{0}^{1, p}(\Omega)\right)$ but does not contain any strongly convergent subsequence in $L^{p}\left(I, L^{q}(\Omega)\right)$. Thus, Theorem 1.3 can not be applied to the operator $\mathcal{A}:=\mathcal{S}+\mathcal{B}$. Nevertheless, it was already observed in [14, Theorems 2.5.1, 3.1.2] that, using and adapting the ideas of the proof of Theorem 1.3, one can show the existence of solutions $\mathbf{u} \in W$ of (1.7) if $p>11 / 5$.

In fact, this situation is prototypical and not an exception. To be more precise, assume that the operator $\mathcal{A}: L^{p}(I, V) \rightarrow\left(L^{p}(I, V)\right)^{*}$ is induced by a family of operators $A(t): V \rightarrow V^{*}, t \in I$. We can not expect $\mathcal{A}: L^{p}(I, V) \rightarrow\left(L^{p}(I, V)\right)^{*}$ to be pseudo-monotone even if the operators $\{A(t)\}_{t \in I}$ are pseudo-monotone. This is due to the fact that $\boldsymbol{x}_{n} \rightarrow \boldsymbol{x}$ in $L^{p}(I, V)$ in general does not imply $\boldsymbol{x}_{n}(t) \rightarrow$ $\boldsymbol{x}(t)$ in $V$ for a.e. $t \in I$ (cf. Remark 2.16). Thus, the pseudo-monotonicity of the operators $A(t)$ can not be used. However, adapting the ideas of [10,11,14] one can show the existence of solutions to the evolutionary problem in many cases (cf. [1] for a treatment using this approach). The drawback of this approach is that additional technical assumptions on the spaces have to be made in order to use the Aubin-Lions lemma. These additional assumptions are not natural and in fact even not needed. It is the first purpose of this paper to prove an abstract existence theorem for evolutionary problems (cf. Theorem 4.1) that avoids unnecessary technical assumptions and is applicable if Theorem 1.3 is not applicable but [14, Theorem 2.5.1] could be adapted. This leads us to the new notion of Bochner pseudo-monotone operators. Moreover, based on the methods in $[1,13,16-18,20]$, we are able to prove that the induced operator $\mathcal{A}$ of a family of pseudo-monotone operators $\{A(t)\}_{t \in I}$ satisfying appropriate coercivity and growth conditions (cf. conditions (C.1)-(C.5) which are easily verifiable

\footnotetext{
${ }^{2}$ The dyadic product $\mathbf{a} \otimes \mathbf{b} \in \mathbb{R}^{3 \times 3}$ of two vectors $\mathbf{a}=\left(a_{1}, a_{2}, a_{3}\right)^{\top}, \mathbf{b}=\left(b_{1}, b_{2}, b_{3}\right)^{\top} \in \mathbb{R}^{3}$ is defined as $(\mathbf{a} \otimes \mathbf{b})_{i j}:=a_{i} b_{j}$ for every $i, j=1,2,3$.

${ }^{3}$ This failure of the compactness is due to the fact that no information of the time derivative has been taken into account. If $\mathcal{B}$ is considered as an operator from $W^{1, p, p^{\prime}}\left(I, V, V^{*}\right)$ to $\left(W^{1, p, p^{\prime}}\left(I, V, V^{*}\right)\right)^{*}$ one can show that $\mathcal{B}$ is compact. However, on these spaces the operator $\mathcal{A}$ is not coercive, and Theorem 1.3 is again not applicable.
} 
in concrete applications) is Bochner pseudo-monotone. Note that the operator $\mathcal{A}=$ $\mathcal{S}+\mathcal{B}$, introduced above for the treatment of problem (1.7), satisfies these conditions. Consequently, Theorem 4.1 or Corollary 4.2 are applicable, in contrast to Theorem 1.3, which is not. This observation applies to many other applications, especially those where the inducing operator contains a compact part. Thus, it seems that Bochner pseudo-monotonicity plays the same role for nonlinear evolution problems as classical pseudo-monotonicity for time-independent nonlinear problems. This is due to the fact that Bochner pseudo-monotonicity takes into account the informations both from the operator and the time derivative. In the same spirit, we introduce the notion of Bochner coercivity, which generalizes the usual coercivity of the operator in the sense that it also takes into account the information coming from the time derivative based on Gronwall's inequality.

Consider now the problem (1.7) without the convective term. In this case Theorem 1.3 is applicable. However, there appears the restriction $p>6 / 5$, since the spaces $V, H$ have to form an evolution triple. This lower bound is artificial and can be avoided if one works on the intersection space $V \cap H$, at least in the case of a monotone operator, as already observed in [14, Theorem 2.1.2bis]. The second purpose of this paper is to avoid artificial lower bounds based on problems with appropriate embeddings. Thus, we introduce the notion of pre-evolution triples, based on pull-back intersections, which generalize evolution triples. Moreover, we develop the abstract theory of Bochner pseudo-monotone operators immediately for pre-evolution triples.

The paper is organized as follows: In Sect. 2, we introduce the notation and some basic definitions and results concerning Bochner-Lebesgue spaces, Bochner-Sobolev spaces, pull-back intersections, evolution equations and introduce the notion of preevolution triples. In Sect. 3, we introduce Bochner pseudo-monotonicity and Bochner coercivity as appropriate extensions of the concepts of pseudo-monotonicity and coercivity to the evolutionary setting. In view of applications we will present some sufficient conditions on operator families that imply these concepts. In Sect. 4, we prove an existence result for evolution equations with pre-evolution triples for abstract Bochner pseudo-monotone and Bochner coercive operators as well as for operators satisfying appropriate and easily verifiable sufficient conditions. Section 5 contains some illustrating examples for the before developed theory.

The paper is an extended and modified version of parts of the thesis [12].

\section{Preliminaries}

\subsection{Operators}

For a Banach space $X$ with norm $\|\cdot\|_{X}$ we denote by $X^{*}$ its dual space equipped with the norm $\|\cdot\|_{X^{*}}$. The duality pairing is denoted by $\langle\cdot, \cdot\rangle_{X}$. All occurring Banach spaces are assumed to be real. By $D(A)$ we denote the domain of definition of an 
operator $A: D(A) \subseteq X \rightarrow Y$, and by $R(A):=\{A x \mid x \in D(A)\}$ its range. The following notions turn out to be useful in our investigation.

Definition 2.1. Let $\left(X,\|\cdot\|_{X}\right)$ and $\left(Y,\|\cdot\|_{Y}\right)$ be Banach spaces. The operator $A$ : $D(A) \subseteq X \rightarrow Y$ is said to be

(i) bounded, if for all bounded $M \subseteq D(A) \subseteq X$ the image $A(M) \subseteq Y$ is bounded.

(ii) coercive, if $Y=X^{*}, D(A)$ is unbounded and $\lim _{\substack{\|x\|_{X} \rightarrow \infty \\ x \in D(A)}} \frac{\langle A x, \bar{x}\rangle_{X}}{\|x\|_{X}}=\infty$.

(iii) pseudo-monotone, if $Y=X^{*}, D(A)=X$ and for each sequence $\left(x_{n}\right)_{n \in \mathbb{N}} \subseteq X$ with

$$
\begin{aligned}
& x_{n} \stackrel{n \rightarrow \infty}{\longrightarrow} x \text { in } X, \\
& \limsup _{n \rightarrow \infty}\left\langle A x_{n}, x_{n}-x\right\rangle_{X} \leq 0
\end{aligned}
$$

it follows that $\langle A x, x-y\rangle_{X} \leq \liminf _{n \rightarrow \infty}\left\langle A x_{n}, x_{n}-y\right\rangle_{X}$ for all $y \in X$.

Note that pseudo-monotonicity together with boundedness compensate for the absence of weak continuity of the nonlinear operator $A$, as for a sequence $\left(x_{n}\right)_{n \in \mathbb{N}} \subseteq X$ satisfying (2.2) and (2.3) it follows that $A x_{n} \stackrel{n \rightarrow \infty}{\rightarrow} A x$ in $X^{*}$. Also note that the conditions (2.2) and (2.3) are natural, since if $\left(x_{n}\right)_{n \in \mathbb{N}} \subseteq X$ is a sequence of appropriate Galerkin approximations of the problem (1.2), then (2.2) is a consequence of the demanded coercivity and (2.3) can be derived directly from the properties of the Galerkin approximation.

\subsection{Pre-evolution triple and pull-back intersections}

Existence results for the initial value problem (1.5) (cf. $[4,8,14,18,19,21,24])$ usually require an evolution triple structure $(V, H, j)$, i.e., $\left(V,\|\cdot\|_{V}\right)$ is a separable, reflexive Banach space, $\left(H,(\cdot, \cdot)_{H}\right)$ a Hilbert space and $j: V \rightarrow H$ an embedding, such that $R(j)$ is dense in $H$, e.g., $V=W_{0}^{1, p}(\Omega), H=L^{2}(\Omega)$ and $j=$ id for $p \geq \frac{2 d}{d+2}$ fulfill these requirements. This evolution triple structure is primarily needed for the validity of an integration by parts formula (cf. Proposition 2.23). Note that especially the existence of a dense embedding in the definition of evolution triples limits the scope of application, since for example $W_{0}^{1, p}(\Omega)$ does not embed into $L^{2}(\Omega)$ if $1<p<\frac{2 d}{d+2}$.

Lions in [14] circumvented this limitation in the case of autonomous monotone operators $A: V \rightarrow V^{*}$ by modifying the coercivity condition $\langle A v, v\rangle_{V} \geq c\|v\|_{V}^{p}$ to $\langle A v, v\rangle_{V} \geq c[v]_{V}^{p}$, where $[\cdot]_{V}$ is a semi-norm on $V$ such that $[v]_{V}+\lambda\|v\|_{H} \geq \beta\|v\|_{V}$ for all $v \in V$ and for some $\lambda, \beta>0$. If $A$ is generated by the $p$-Laplace operator for $p \in\left(1, \frac{2 d}{d+2}\right)$ the above situation is realized by $V=W_{0}^{1, p}(\Omega) \cap L^{2}(\Omega)$, equipped with $\|\cdot\|_{V}=\|\cdot\|_{W_{0}^{1, p}(\Omega)}+\|\cdot\|_{L^{2}(\Omega)}, H=L^{2}(\Omega)$ and $[\cdot]_{V}=\|\cdot\|_{W_{0}^{1, p}(\Omega)}$.

We proceed similarly and consider a separable, reflexive Banach space $V$, a Hilbert space $H$ such that $V \cap H$ exists. We equip $V \cap H$ with the canonical sum norm $\|\cdot\|_{V}=\|\cdot\|_{V}+\|\cdot\|_{H}$, such that trivially $V \cap H$ embeds into $H$ densely, i.e., 
$V \cap H$ and $H$ form an evolution triple. Moreover, we relax the coercivity condition further, since we take into account the information coming from the time derivative. To make this precise (cf. Definition 2.10) we need some facts on intersections of Banach spaces. To this end, we want to propose an alternative point of view, which turns out to be both quite comfortable and exact, in the sense that we do not need to assume any identifications of spaces and the amount of occurring embeddings is marginal. Nonetheless, we emphasize that the standard definition of intersections of Banach spaces (cf. [2]) is equivalent to our approach and all the following assertions remain true if we use the standard framework in [2].

Definition 2.4. (Embedding) Let $\left(X, \tau_{X}\right)$ and $\left(Y, \tau_{Y}\right)$ be topological vector spaces. The operator $j: X \rightarrow Y$ is said to be an embedding if it is linear, injective and continuous. In this case we use the notation

$$
X \stackrel{j}{\hookrightarrow} Y .
$$

If $X \subseteq Y$ and $j=\operatorname{id}_{X}: X \rightarrow Y$, then we write $X \hookrightarrow Y$ instead.

Definition 2.5. (Compatible couple) Let $\left(X,\|\cdot\|_{X}\right)$ and $\left(Y,\|\cdot\|_{Y}\right)$ be Banach spaces such that embeddings $e_{X}: X \rightarrow Z$ and $e_{Y}: Y \rightarrow Z$ into a Hausdorff vector space $\left(Z, \tau_{Z}\right)$ exist. Then, we call $(X, Y):=\left(X, Y, Z, e_{X}, e_{Y}\right)$ a compatible couple.

Definition 2.6. (Pull-back intersection of Banach spaces) Let $(X, Y)$ be a compatible couple. Then, the operator $j:=e_{Y}^{-1} e_{X}: e_{X}^{-1}\left(R\left(e_{X}\right) \cap R\left(e_{Y}\right)\right) \rightarrow Y$ is well-defined and we denote by

$$
X \cap_{j} Y:=e_{X}^{-1}\left(R\left(e_{X}\right) \cap R\left(e_{Y}\right)\right) \subseteq X
$$

the pull-back intersection of $X$ and $Y$ in $X$ with respect to $j$. Furthermore, $j$ is said to be the corresponding intersection embedding. If $X, Y \subseteq Z$ with $e_{X}=\operatorname{id}_{X}$ and $e_{Y}=\operatorname{id}_{Y}$, we set $X \cap Y:=X \cap_{j} Y$.

The next proposition shows that $j: X \cap_{j} Y \rightarrow Y$ is indeed an embedding if $X \cap_{j} Y$ is equipped with an appropriate norm.

Proposition 2.7. Let $(X, Y)$ be a compatible couple. Then, $X \cap_{j} Y$ is a vector space and equipped with norm

$$
\|\cdot\|_{X \cap_{j} Y}:=\|\cdot\|_{X}+\|j \cdot\|_{Y}
$$

a Banach-space. Moreover, $j: X \cap_{j} Y \rightarrow Y$ is an embedding.

Proof. In [2, Chapter 3, Theorem 1.3] it is proved that $R\left(e_{X}\right) \cap R\left(e_{Y}\right)$ equipped with the norm $\|\cdot\|_{R\left(e_{X}\right) \cap R\left(e_{Y}\right)}:=\left\|e_{X}^{-1}(\cdot)\right\|_{X}+\left\|e_{Y}^{-1}(\cdot)\right\|_{Y}$ is a Banach space. Since $e_{X}^{-1}: R\left(e_{X}\right) \cap R\left(e_{Y}\right) \rightarrow X \cap_{j} Y$ is an isometry, if we equip $X \cap_{j} Y$ with the norm $\|\cdot\|_{X \cap_{j} Y}$, the space $X \cap_{j} Y$ inherits the Banach space property of $R\left(e_{X}\right) \cap R\left(e_{Y}\right)$. The linearity and injectivity of $j: X \cap_{j} Y \rightarrow Y$ are clear. The continuity of $j: X \cap_{j} Y \rightarrow Y$ follows directly from the definition of the norm in $X \cap_{j} Y$. 


\section{Remark 2.8. (Fundamental properties of pull-back intersections)}

(i) Consistency: If $\left(X,\|\cdot\|_{X}\right)$ and $\left(Y,\|\cdot\|_{Y}\right)$ are Banach spaces such that an embedding $j: X \rightarrow Y$ exists, then $(X, Y)=\left(X, Y, Y, j, \operatorname{id}_{Y}\right)$ forms a compatible couple and it holds $X \cap_{j} Y=X$ with norm equivalence $\|\cdot\|_{X \cap_{j} Y} \sim\|\cdot\| \|_{X}$.

(ii) Commutativity up to isomorphism: For a compatible couple $(X, Y)$ the pullback intersection $Y \cap_{j^{-1}} X$ of $X$ and $Y$ in $Y$ with respect to $j^{-1}=e_{X}^{-1} e_{Y}$ is well-defined as well. In addition, $j: X \cap_{j} Y \rightarrow Y \cap_{j^{-1}} X$ is an isometric isomorphism. Rephrased, pull-back intersections are thus commutative up to an isometric isomorphism.

(iii) Associativity: If $(X, Y)=\left(X, Y, Z, e_{X}, e_{Y}\right)$ and $(Y, W)=\left(Y, W, Z, e_{Y}, e_{W}\right)$ are compatible couples, $i:=e_{W}^{-1} e_{X}, j:=e_{Y}^{-1} e_{X}$ and $k:=e_{W}^{-1} e_{Y}$, then it holds $\left(X \cap_{j} Y\right) \cap_{i} W=X \cap_{j}\left(Y \cap_{k} W\right)$ and $\|\cdot\|_{\left(X \cap_{j} Y\right) \cap_{i} W}=\|\cdot\|_{X \cap_{j}\left(Y \cap_{k} W\right)}$.

Proposition 2.9. Let $(X, Y)$ be a compatible couple. Then, it holds:

(i) The graph $G(j):=\left\{(x, y)^{\top} \in X \times Y \mid x \in X \cap_{j} Y, y=j x\right\}$ is closed in $X \times Y$.

(ii) If $X$ and $Y$ are reflexive or separable, then $X \cap_{j} Y$ is as well.

(iii) First characterization of weak convergence in $X \cap_{j} Y:$ A sequence $\left(x_{n}\right)_{n \in \mathbb{N}} \subset$ $X \cap_{j} Y$ and $x \in X \cap_{j} Y$ satisfy $x_{n} \stackrel{n \rightarrow \infty}{\rightarrow} x$ in $X \cap_{j} Y$ if and only if $x_{n} \stackrel{n \rightarrow \infty}{\rightarrow} x$ in $X$ and $j x_{n} \stackrel{n \rightarrow \infty}{\rightarrow} j x$ in $Y$.

(iv) Second characterization of weak convergence in $X \cap_{j} Y$ : In addition let $X$ be reflexive. A sequence $\left(x_{n}\right)_{n \in \mathbb{N}} \subset X \cap_{j} Y$ and $x \in X \cap_{j} Y$ satisfy $x_{n} \stackrel{n \rightarrow \infty}{\longrightarrow}$ $x$ in $X \cap_{j} Y$ if and only if $\sup _{n \in \mathbb{N}}\left\|x_{n}\right\|_{X}<\infty$ and $j x_{n} \stackrel{n \rightarrow \infty}{\rightarrow} j x$ in $Y$.

Proof. ad (i) Let $X \times Y$ be the product of $X$ and $Y$, which equipped with norm $\left\|(x, y)^{\top}\right\|_{X \times Y}:=\|x\|_{X}+\|y\|_{Y}$ is a Banach space. Hence,

$$
L: X \cap_{j} Y \rightarrow G(j) \subseteq X \times Y: x \mapsto(x, j x)^{\top}
$$

is a linear isometric isomorphism of $X \cap_{j} Y$ onto $G(j)$. Therefore, $G(j)$ is closed in $X \times Y$.

ad (ii): If $X$ and $Y$ are reflexive, then also $X \times Y$ is reflexive. Since $G(j)$ is closed in $X \times Y$, it is also reflexive. As $L$ is an isomorphism we finally transfer the reflexivity from $G(j)$ to $X \cap_{j} Y$. If $X$ and $Y$ are separable, then also $X \times Y$ is separable and $G(j)$ as well. As $L$ is an isometric isomorphism $X \cap_{j} Y$ has to be separable as well.

ad (iii): Follows from the fact that weak convergence in $X \times Y$ is characterized by weak convergence of all components in conjunction with the isometric isomorphism $L$.

ad (iv):

The necessity follows immediately from (iii). To prove the sufficiency let $x \in X \cap_{j} Y$ and $\left(x_{n}\right)_{n \in \mathbb{N}} \subset X \cap_{j} Y$ satisfy $\sup _{n \in \mathbb{N}}\left\|x_{n}\right\|_{X}<\infty$ and $j x_{n} \stackrel{n \rightarrow \infty}{\rightarrow} j x$ in $Y$. Let $\left(x_{n}\right)_{n \in \Lambda} \subseteq X \cap_{j} Y$ with $\Lambda \subseteq \mathbb{N}$ be an arbitrary subsequence. In particular, $\left(x_{n}\right)_{n \in \Lambda} \subseteq$ 
$X$ is bounded. Then, Eberlein-Šmuljan's theorem yields the existence of both a subsequence $\left(x_{n}\right)_{n \in \Lambda_{1}} \subseteq X \cap_{j} Y$ with $\Lambda_{1} \subseteq \Lambda$ and an element $\tilde{x} \in X$ such that

$$
x_{n} \stackrel{n \rightarrow \infty}{\rightarrow} \tilde{x} \quad \text { in } X\left(n \in \Lambda_{1}\right) .
$$

We have $\left(\left(x_{n}, j x_{n}\right)^{\top}\right)_{n \in \Lambda_{1}} \subseteq G(j)$. As weak convergence of all components implies weak convergence in the corresponding Cartesian product we obtain

$$
\left(x_{n}, j x_{n}\right)^{\top} \stackrel{n \rightarrow \infty}{\longrightarrow}(\tilde{x}, j x)^{\top} \quad \text { in } X \times Y\left(n \in \Lambda_{1}\right) .
$$

$G(j)$ is weakly closed in $X \times Y$, as it is convex and closed in $X \times Y$ (cf. Proposition 2.9). In consequence, it holds $(\tilde{x}, j x)^{\top} \in G(j)$, i.e., $\tilde{x} \in X \cap_{j} Y$ and $j x=j \tilde{x}$ in $Y$. From the injectivity of $j: X \cap_{j} Y \rightarrow Y$ we deduce further that $x=\tilde{x}$ in $X \cap_{j} Y$. Thus, the first characterization of weak convergence in pull-back intersections provides

$$
x_{n} \stackrel{n \rightarrow \infty}{\rightarrow} x \quad \text { in } X \cap_{j} Y\left(n \in \Lambda_{1}\right) .
$$

Hence, $x \in X \cap_{j} Y$ is weak accumulation point of each subsequence of $\left(x_{n}\right)_{n \in \mathbb{N}} \subseteq$ $X \cap_{j} Y$. The standard convergence principle (cf. [8, Kap. I, Lemma 5.4]) yields $x_{n} \stackrel{n \rightarrow \infty}{\longrightarrow}$ $x$ in $X \cap_{j} Y$.

Now, we have created an appropriate framework to give a detailed description of the concept of pre-evolution triples.

Definition 2.10. (Pre-evolution triple) Let $(V, H):=\left(V, H, Z, e_{V}, e_{H}\right)$ be a compatible couple, $\left(V,\|\cdot\|_{V}\right)$ a separable, reflexive Banach space and $\left(H,(\cdot, \cdot)_{H}\right)$ a separable Hilbert space. In this situation the pull-back intersection of $V$ and $H$ is defined as $V \cap_{j} H:=e_{V}^{-1}\left(R\left(e_{V}\right) \cap R\left(e_{H}\right)\right)$, and the intersection embedding is defined as $j:=e_{H}^{-1} e_{V}: V \cap_{j} H \rightarrow H$. If

$$
\overline{R(j)}^{\|\cdot\|_{H}}={\overline{j\left(V \cap_{j} H\right)}}^{\|\cdot\|_{H}}=H,
$$

then the triple $(V, H, j)$ is said to be a pre-evolution triple. Let $R: H \rightarrow H^{*}$ be the Riesz isomorphism with respect to $(\cdot, \cdot)_{H}$. As $j$ is a dense embedding the adjoint $j^{*}: H^{*} \rightarrow\left(V \cap_{j} H\right)^{*}$ and therefore $e:=j^{*} R j: V \cap_{j} H \rightarrow\left(V \cap_{j} H\right)^{*}$ are embeddings as well. We call $e$ the canonical embedding of $(V, H, j)$. Note that

$$
\langle e v, w\rangle_{V \cap_{j} H}=(j v, j w)_{H} \quad \text { for all } v, w \in V \cap_{j} H .
$$

Remark 2.12. The notion of a pre-evolution triple generalizes the standard notion of an evolution triple. Note that an evolution triple is a pre-evolution triple, since $\left(V, H, H, j, \mathrm{id}_{H}\right)$ is a compatible couple. Moreover, the intersection embedding is the embedding $j$, and we have $V=V \cap_{j} H$ with norm equivalence $\|\cdot\|_{V} \sim \|$. $\|_{V \cap_{j} H}$. Thus, if the pre-evolution triple is an evolution triple we can just replace the intersection $V \cap_{j} H$ by $V$. On the other hand, if $(V, H, j)$ is a pre-evolution triple, then $\left(V \cap_{j} H, H, j\right)$ is an evolution triple. 


\subsection{Bochner-Lebesgue spaces}

In this paragraph we collect some well-known results concerning Bochner-Lebesgue spaces, which will be used in the following. By $\left(X,\|\cdot\|_{X}\right)$ and $\left(Y,\|\cdot\|_{Y}\right)$ we always denote Banach spaces, by $I:=(0, T)$, with $0<T<\infty$, a finite time interval and by $\mathcal{M}(I, X)$ the vector space of Bochner measurable functions from $I$ into $X$.

Proposition 2.13. Let $\left(X,\|\cdot\|_{X}\right)$ be a reflexive Banach space and $1<p<\infty$. Then, the operator $R: L^{p^{\prime}}\left(I, X^{*}\right) \rightarrow\left(L^{p}(I, X)\right)^{*}$, for every $f \in L^{p^{\prime}}\left(I, X^{*}\right)$ and $\boldsymbol{x} \in L^{p}(I, X)$ given via

$$
\langle R \boldsymbol{f}, \boldsymbol{x}\rangle_{L^{p}(I, X)}:=\int_{I}\langle\boldsymbol{f}(t), \boldsymbol{x}(t)\rangle_{X} d t,
$$

is an isometric isomorphism. In particular, $L^{p}(I, X)$ is reflexive.

Proof. See [7, IV. 1. Theorem 1, Corollary 2].

Proposition 2.14. Let $\boldsymbol{x}: I \rightarrow X$ be a function such that there exists a sequence $\left(\boldsymbol{x}_{n}\right)_{n \in \mathbb{N}} \subseteq \mathcal{M}(I, X)$ with

$$
\boldsymbol{x}_{n}(t) \stackrel{n \rightarrow \infty}{\rightarrow} \boldsymbol{x}(t) \text { in } X
$$

for almost every $t \in I$. Then, $\boldsymbol{x} \in \mathcal{M}(I, X)$.

Proof. We apply Pettis' theorem (cf. [22, Chapter V, Theorem: (Pettis)]) to obtain Lebesgue measurable sets $N_{n} \subseteq I, n \in \mathbb{N}$, such that $I \backslash N_{n}$ are null sets and $\boldsymbol{x}_{n}\left(N_{n}\right)$ are separable. Thus, if we replace $X$ by the closure of $\operatorname{span}\left\{\bigcup_{n \in \mathbb{N}} \boldsymbol{x}_{n}\left(N_{n}\right)\right\}$, it turns out that it suffices to treat the case of separable $X$. For a proof of the latter one we refer to [19, Folgerung 1.10].

Proposition 2.15. Let $\left(X,\|\cdot\|_{X}\right)$ be a reflexive Banach space and $1<p<\infty$. If the sequence $\left(\boldsymbol{x}_{n}\right)_{n \in \mathbb{N}} \subseteq L^{p}(I, X)$ is bounded and satisfies

$$
\boldsymbol{x}_{n}(t) \stackrel{n \rightarrow \infty}{\rightarrow} \boldsymbol{x}(t) \text { in } X
$$

for almost every $t \in I$, then $\boldsymbol{x}_{n} \stackrel{n \rightarrow \infty}{\rightarrow} \boldsymbol{x}$ in $L^{p}(I, X)$.

Proof. It suffices to treat the case $\boldsymbol{x}=\mathbf{0}$ in $L^{p}(I, X)$. For arbitrary $\boldsymbol{x}^{*} \in\left(L^{p}(I, X)\right)^{*}$ we get $\left\langle\left(R^{-1} x^{*}\right)(t), x_{n}(t)\right\rangle_{X} \rightarrow 0(n \rightarrow \infty)$ for almost every $t \in I$. In particular, for Lebesgue measurable $E \subseteq I$ we obtain

$$
\int_{E}\left|\left\langle\left(R^{-1} \boldsymbol{x}^{*}\right)(s), \boldsymbol{x}_{n}(s)\right\rangle_{X}\right| \mathrm{d} s \leq\left\|R^{-1} \boldsymbol{x}^{*} \chi_{E}\right\|_{L^{p^{\prime}}\left(I, X^{*}\right)}\left\|\boldsymbol{x}_{n}\right\|_{L^{p}(I, X)} \leq c\left\|R^{-1} \boldsymbol{x}^{*} \chi_{E}\right\|_{L^{p^{\prime}\left(I, X^{*}\right)}},
$$

where we exploited the boundedness of the sequence $\left(\boldsymbol{x}_{n}\right)_{n \in \mathbb{N}} \subseteq L^{p}(I, X)$. Therefore, the sequence $\left(\left\langle\left(R^{-1} \boldsymbol{x}^{*}\right)(\cdot), \boldsymbol{x}_{n}(\cdot)\right\rangle_{X}\right)_{n \in \mathbb{N}} \subseteq L^{1}(I)$ is uniformly integrable and Vitali's theorem eventually yields $\left\langle\boldsymbol{x}^{*}, \boldsymbol{x}_{n}\right\rangle_{L^{p}(I, X)}=\int_{I}\left\langle\left(R^{-1} \boldsymbol{x}^{*}\right)(t), \boldsymbol{x}_{n}(t)\right\rangle_{X} \mathrm{~d} t \rightarrow 0(n \rightarrow$ $\infty)$. 
Remark 2.16. The converse implication in Proposition 2.15 is in general not true. This can be seen by the following easy example. Let $I=(0,2 \pi), p \in(1, \infty), X=\mathbb{R}$ and $\left(\boldsymbol{x}_{n}\right)_{n \in \mathbb{N}} \subseteq L^{\infty}(I, \mathbb{R})$, given via $\boldsymbol{x}_{n}(t)=\sin (n t)$ for every $t \in I$ and all $n \in \mathbb{N}$. Then, there holds $\boldsymbol{x}_{n} \rightarrow \mathbf{0}$ in $L^{p}(I, \mathbb{R})(n \rightarrow \infty)$, but not $\boldsymbol{x}_{n}(t) \rightarrow 0$ for almost every $t \in I$ $(n \rightarrow \infty)$.

Definition 2.17. (Induced mapping) Let $A(t): X \rightarrow Y, t \in I$, be a family of operators with the following properties:

(C.1) $A(t): X \rightarrow Y$ is demi-continuous for almost every $t \in I$.

(C.2) $A(\cdot) x: I \rightarrow Y$ is Bochner measurable for all $x \in X$.

Then, we define the induced mapping $A: \mathcal{M}(I, X) \rightarrow \mathcal{M}(I, Y)$ of $\{A(t)\}_{t \in I}$ by

$$
(\boldsymbol{A x})(t):=A(t)(\boldsymbol{x}(t)) \text { in } Y
$$

for almost every $t \in I$ and all $\boldsymbol{x} \in \mathcal{M}(I, X)$.

In the case $X=V$ and $Y=V^{*}$, where $(V, H, j)$ is an evolution triple, the welldefinedness of the induced operator is proved in [24]. The next lemma treats the general case.

Lemma 2.18. Let $A(t): X \rightarrow Y, t \in I$, be a family of operators satisfying $(C .1)$ and (C.2). Then, the induced mapping $A: \mathcal{M}(I, X) \rightarrow \mathcal{M}(I, Y)$ is well-defined.

Proof. For $\boldsymbol{x} \in \mathcal{M}(I, X)$ there exists a sequence of simple functions $\left(s_{n}\right)_{n \in \mathbb{N}} \subseteq$ $\mathcal{S}(I, X)$, i.e., $s_{n}(t)=\sum_{i=1}^{k_{n}} s_{i}^{n} \chi_{E_{i}^{n}}(t)$, where $s_{i}^{n} \in X, k_{n} \in \mathbb{N}$ and $E_{i}^{n} \in \mathcal{L}^{1}(I)$ with $E_{i}^{n} \cap E_{j}^{n}=\emptyset$ for $i \neq j$, which is converging almost everywhere to $\boldsymbol{x}(t)$ in $X$. Due to (C.2) $\mathbf{A} s_{i}^{n}=A(\cdot) s_{i}^{n}: I \rightarrow Y$ and therefore also

$$
\boldsymbol{A} \boldsymbol{s}_{n}=\sum_{i=1}^{k_{n}} \chi_{E_{i}^{n}} \boldsymbol{A} s_{i}^{n}+\chi_{\left\{I \backslash \bigcup_{i=1}^{k_{n}} E_{i}^{n}\right\}} \boldsymbol{A 0 : I \rightarrow Y}
$$

are Bochner measurable and converge almost everywhere weakly to $(\boldsymbol{A x})(t)$ in $Y$ due to (C.1). Thus, Proposition 2.14 ensures $\boldsymbol{A x} \in \mathcal{M}(I, Y)$.

The next proposition shows that embeddings between Banach spaces transmit their properties to the induced operator between Bochner-Lebesgue spaces.

Proposition 2.19. Let $1 \leq p \leq \infty$ and let $e: X \rightarrow Y$ be an embedding. Then, the induced embedding $\boldsymbol{e}: L^{p}(I, X) \rightarrow L^{p}(I, Y)$, for every $\boldsymbol{x} \in L^{p}(I, X)$ given via $(\boldsymbol{e x})(t):=e(\boldsymbol{x}(t))$ in $Y$ for almost every $t \in I$, is well-defined and an embedding.

Proof. Concerning the well-definedness, linearity and boundedness we refer to [22, Chapter V, 5. Bochner's Integral, Corollary 2]. The verification of the injectivity is elementary and thus omitted.

The next remark examines how the concept of pull-back intersections transfer to the Bochner-Lebesgue level. 
Remark 2.20. (Induced compatible couple) Let $(X, Y)=\left(X, Y, Z, e_{X}, e_{Y}\right)$ be a compatible couple (cf. Definition 2.5) and $1 \leq p, q \leq \infty$. In [2, Chapter 3, Theorem 1.3] it is proved that the sum $R\left(e_{X}\right)+R\left(e_{Y}\right) \subseteq Z$ equipped with the norm

$$
\|z\|_{R\left(e_{X}\right)+R\left(e_{Y}\right)}:=\inf _{\substack{x \in X, y \in Y \\ z=e_{X} x+e_{Y} y}} \max \left\{\|x\|_{X},\|y\|_{Y}\right\}
$$

is a Banach space. Then, both $e_{X}: X \rightarrow R\left(e_{X}\right)+R\left(e_{Y}\right)$ and $e_{Y}: Y \rightarrow R\left(e_{X}\right)+R\left(e_{Y}\right)$ are embeddings (cf. Definition 2.4) and therefore due to Proposition 2.19 also the induced operators

$$
\begin{aligned}
& \boldsymbol{e}_{X}: L^{p}(I, X) \rightarrow L^{1}\left(I, R\left(e_{X}\right)+R\left(e_{Y}\right)\right) \text { given via }\left(\boldsymbol{e}_{X} \boldsymbol{x}\right)(t):=e_{X}(\boldsymbol{x}(t)) \text { for a.e. } t \in I, \\
& \boldsymbol{e}_{Y}: L^{q}(I, Y) \rightarrow L^{1}\left(I, R\left(e_{X}\right)+R\left(e_{Y}\right)\right) \text { given via }\left(\boldsymbol{e}_{Y} \boldsymbol{y}\right)(t):=e_{Y}(\boldsymbol{y}(t)) \text { for a.e. } t \in I
\end{aligned}
$$

Consequently,

$$
\left(L^{p}(I, X), L^{q}(I, Y)\right)=\left(L^{p}(I, X), L^{q}(I, Y), L^{1}\left(I, R\left(e_{X}\right)+R\left(e_{Y}\right)\right), \boldsymbol{e}_{X}, \boldsymbol{e}_{Y}\right)
$$

is a compatible couple. In accordance with Definition 2.6, the pull-back intersection

$$
L^{p}(I, X) \cap_{j} L^{q}(I, Y)
$$

where $\boldsymbol{j}:=\boldsymbol{e}_{Y}^{-1} \boldsymbol{e}_{X}$, and the corresponding intersection embedding

$$
\boldsymbol{j}: L^{p}(I, X) \cap_{j} L^{q}(I, Y) \rightarrow L^{q}(I, Y)
$$

is well-defined.

Next we give an alternative representation of pull-back intersections of BochnerLebesgue spaces, from which we are able to deduce Bochner measurability with respect to $X \cap_{j} Y$ directly.

Proposition 2.21. Let $(X, Y)$ be a compatible couple and $1 \leq p \leq q \leq \infty$. Then,

$$
\left(L^{p}\left(I, X \cap_{j} Y\right), L^{q}(I, Y)\right)=\left(L^{p}\left(I, X \cap_{j} Y\right), L^{q}(I, Y), L^{p}(I, Y), j, i d_{L^{q}(I, Y)}\right)
$$

is a compatible couple, where $j$ is defined in Remark 2.20. Thus, $L^{p}(I, X \cap j Y) \cap_{j}$ $L^{q}(I, Y)$ and $j: L^{p}(I, X \cap j Y) \cap_{j} L^{q}(I, Y) \rightarrow L^{q}(I, Y)$ are well-defined. In particular, it holds

$$
L^{p}\left(I, X \cap_{j} Y\right) \cap_{j} L^{q}(I, Y)=L^{p}(I, X) \cap_{j} L^{q}(I, Y)
$$

with norm equivalence.

Proof. As $j: X \cap_{j} Y \rightarrow Y$ is an embedding, the induced operator $j: L^{p}\left(I, X \cap_{j}\right.$ $Y) \rightarrow L^{p}(I, Y)$ is an embedding as well, due to Proposition 2.19. Therefore,

$$
\left(L^{p}\left(I, X \cap_{j} Y\right), L^{q}(I, Y)\right)=\left(L^{p}\left(I, X \cap_{j} Y\right), L^{q}(I, Y), L^{p}(I, Y), j, \operatorname{id}_{L^{q}(I, Y)}\right)
$$


is a compatible couple, and $L^{p}\left(I, X \cap_{j} Y\right) \cap_{j} L^{q}(I, Y)$ and $j: L^{p}\left(I, X \cap_{j} Y\right) \cap_{j}$ $L^{q}(I, Y) \rightarrow L^{q}(I, Y)$ are well-defined. Proposition 2.19 also implies $\left(j^{-1} \boldsymbol{y}\right)(t)=$ $j^{-1}(\boldsymbol{y}(t))=e_{X}^{-1} e_{Y}(\boldsymbol{y}(t))=\left(\boldsymbol{e}_{X}^{-1} \boldsymbol{e}_{Y} \boldsymbol{y}\right)(t)$ for almost every $t \in I$ and all $\boldsymbol{y} \in R(\boldsymbol{j})=$ $R\left(\boldsymbol{e}_{Y}^{-1} \boldsymbol{e}_{X}\right)$, i.e., $\boldsymbol{j}^{-1}=\boldsymbol{e}_{X}^{-1} \boldsymbol{e}_{Y}$ on $R(\boldsymbol{j})$. From the latter and Definition 2.6 we obtain

$$
\begin{aligned}
L^{p}\left(I, X \cap \cap_{j} Y\right) \cap_{j} L^{q}(I, Y) & =\boldsymbol{j}^{-1}\left(R(\boldsymbol{j}) \cap L^{q}(I, Y)\right) \\
& =\boldsymbol{e}_{X}^{-1} \boldsymbol{e}_{Y}\left(R(\boldsymbol{j}) \cap L^{q}(I, Y)\right) \\
& =\boldsymbol{e}_{X}^{-1}\left(R\left(\boldsymbol{e}_{Y} \boldsymbol{j}\right) \cap R\left(\boldsymbol{e}_{Y} \operatorname{id}_{L^{q}(I, Y)}\right)\right) \\
& =\boldsymbol{e}_{X}^{-1}\left(R\left(\boldsymbol{e}_{X}\right) \cap R\left(\boldsymbol{e}_{Y} \operatorname{id}_{L^{q}(I, Y)}\right)\right) \\
& =L^{p}(I, X) \cap \boldsymbol{j} L^{q}(I, Y) .
\end{aligned}
$$

The verification of the stated norm equivalence is an elementary calculation and thus omitted.

2.4. Evolution equations

For a pre-evolution triple $(V, H, j), I:=(0, T)$, with $0<T<\infty$, and $1<p<\infty$ we set

$$
\mathcal{X}:=L^{p}\left(I, V \cap_{j} H\right), \quad \mathcal{Y}:=L^{\infty}(I, H) .
$$

Definition 2.22. Let $(V, H, j)$ be a pre-evolution triple and $1<p<\infty$. A function $\boldsymbol{x} \in \mathcal{X}$ possesses a generalized time derivative with respect to the canonical embedding $e$ of $(V, H, j)$ if there exists a function $w \in L^{p^{\prime}}\left(I,(V \cap j H)^{*}\right)$ such that

$$
-\int_{I}(j(\boldsymbol{x}(s)), j v)_{H} \varphi^{\prime}(s) \mathrm{d} s=\int_{I}\langle\boldsymbol{w}(s), v\rangle_{V \cap_{j} H} \varphi(s) \mathrm{d} s .
$$

for all $v \in V \cap_{j} H$ and $\varphi \in C_{0}^{\infty}(I)$. As this function $w \in L^{p^{\prime}}\left(I,\left(V \cap_{j} H\right)^{*}\right)$ is unique (cf. [23, Proposition 23.18]), $\frac{d_{e} x}{\mathrm{~d} t}:=w$ is well-defined. By

$\mathcal{W}:=W_{e}^{1, p, p^{\prime}}\left(I, V \cap_{j} H,\left(V \cap_{j} H\right)^{*}\right):=\left\{x \in L^{p}\left(I, V \cap_{j} H\right) \mid \exists \frac{d_{e} x}{\mathrm{~d} t} \in L^{p^{\prime}}\left(I,\left(V \cap_{j} H\right)^{*}\right)\right\}$

we denote the Bochner-Sobolev space with respect to $e$.

Proposition 2.23. Let $(V, H, j)$ be a pre-evolution triple and $1<p<\infty$. Then, it holds:

(i) The space $\mathcal{W}$ forms a Banach space equipped with the norm

$$
\|\cdot\| \mathcal{W}:=\|\cdot\| \mathcal{X}+\left\|\frac{d_{e}}{d t} \cdot\right\| \|_{L^{p}\left(I,\left(V \cap_{j} H\right)^{*}\right)} .
$$

(ii) Given $\boldsymbol{x} \in \mathcal{W}$ the function $\boldsymbol{j} \boldsymbol{x} \in L^{p}(I, H)$, given via $(\boldsymbol{j} \boldsymbol{x})(t):=j(\boldsymbol{x}(t))$ for almost every $t \in I$, possesses a unique representation in $j_{c} x \in C^{0}(\bar{I}, H)$ and the resulting mapping $\boldsymbol{j}_{c}: \mathcal{W} \rightarrow C^{0}(\bar{I}, H)$ is an embedding. 
(iii) Generalized integration by parts formula: It holds

$$
\begin{aligned}
& \int_{t^{\prime}}^{t}\left\langle\frac{d_{e} \boldsymbol{x}}{d t}(s), \boldsymbol{y}(s)\right\rangle_{V \cap_{j} H} d s=\left[\left(\left(\boldsymbol{j}_{c} \boldsymbol{x}\right)(s),\left(\boldsymbol{j}_{c} \boldsymbol{y}\right)(s)\right)_{H}\right]_{s=t^{\prime}}^{s=t}-\int_{t^{\prime}}^{t}\left\langle\frac{d_{e} \boldsymbol{y}}{d t}(s), \boldsymbol{x}(s)\right\rangle_{V \cap_{j} H} d s \\
& \quad \text { for all } \boldsymbol{x}, \boldsymbol{y} \in \mathcal{W} \text { and } t, t^{\prime} \in \bar{I} \text { with } t^{\prime} \leq t .
\end{aligned}
$$

Proof. A straightforward adaption of [23, Proposition 23.23], since $(V \cap j H, H, j)$ is an evolution triple.

For a pre-evolution triple $(V, H, j), I:=(0, T)$, with $0<T<\infty$, and $1<$ $p<\infty$ we call an operator $\mathcal{A}: \mathcal{X} \cap j \mathcal{Y} \rightarrow \mathcal{X}^{*}$ induced by a family of operators $A(t): V \cap_{j} H \rightarrow\left(V \cap_{j} H\right)^{*}, t \in I$, if

$$
\langle\mathcal{A} \boldsymbol{x}, \boldsymbol{y}\rangle_{\mathcal{X}}:=\int_{I}\langle A(t)(\boldsymbol{x}(t)), \boldsymbol{y}(t)\rangle_{V \cap_{j} H} \mathrm{~d} t=\langle R \boldsymbol{A} \boldsymbol{x}, \boldsymbol{y}\rangle_{\mathcal{X}}
$$

for every $\boldsymbol{x} \in \mathcal{X} \cap \boldsymbol{j} \mathcal{Y}$ and $\boldsymbol{y} \in \mathcal{X}$. In particular, (2.24) says that $\mathcal{A}=R \boldsymbol{A}$, i.e., an induced operator can be represented by an induced mapping in virtue of the Riesz isomorphism (cf. Proposition 2.13).

Definition 2.25. (Evolution equation) Let $(V, H, j)$ be a pre-evolution triple and $1<p<\infty$. Moreover, let $\boldsymbol{y}_{0} \in H$ be an initial value, $\boldsymbol{f} \in L^{p^{\prime}}\left(I,(V \cap j H)^{*}\right)$ a right-hand side and $\mathcal{A}: \mathcal{X} \cap j \mathcal{Y} \rightarrow \mathcal{X}^{*}$ an operator induced by a family of operators $A(t): V \cap_{j} H \rightarrow\left(V \cap_{j} H\right)^{*}, t \in I$. Then, the initial value problem

$$
\begin{gathered}
\int_{I}\left\langle\frac{d_{e} \boldsymbol{y}}{\mathrm{d} t}(t), \boldsymbol{\varphi}(t)\right\rangle_{V \cap_{j} H} \mathrm{~d} t+\int_{I}\langle A(t)(\boldsymbol{y}(t)), \boldsymbol{\varphi}(t)\rangle_{V \cap_{j} H} \mathrm{~d} t=\int_{I}\langle\boldsymbol{f}(t), \boldsymbol{\varphi}(t)\rangle_{V \cap_{j} H} \mathrm{~d} t \quad \forall \boldsymbol{\varphi} \in \boldsymbol{\mathcal { X }}, \\
\left(\boldsymbol{j}_{C} \boldsymbol{y}\right)(0)=\boldsymbol{y}_{0} \quad \text { in } H
\end{gathered}
$$

is said to be an evolution equation. The initial condition has to be understood in the sense of the unique continuous representation $\boldsymbol{j}_{c} \boldsymbol{y} \in C^{0}(\bar{I}, H)$ (cf. Proposition 2.23 (ii)).

\section{Bochner pseudo-monotonicity and Bochner coercivity}

In this section, we introduce the notions Bochner pseudo-monotonicity and Bochner coercivity.

Definition 3.1. (Bochner pseudo-monotonicity) Let $(V, H, j)$ be a pre-evolution triple and $1<p<\infty$. An operator $\mathcal{A}: D(\mathcal{A}) \subseteq \mathcal{X} \rightarrow \mathcal{X}^{*}$ with $D(\mathcal{A}) \supseteq \mathcal{X} \cap_{j} \mathcal{Y}$ is said to be Bochner pseudo-monotone if for a sequence $\left(x_{n}\right)_{n \in \mathbb{N}} \subseteq \mathcal{X} \cap_{j} \mathcal{Y}$ from

$$
\begin{array}{cl}
\boldsymbol{x}_{n} \stackrel{n \rightarrow \infty}{\rightarrow} \boldsymbol{x} & \text { in } \mathcal{X}, \\
\boldsymbol{j} \boldsymbol{x}_{n} \stackrel{*}{\rightarrow} \boldsymbol{j} \boldsymbol{x} & \text { in } \mathcal{Y} \quad(n \rightarrow \infty), \\
\left(\boldsymbol{j} \boldsymbol{x}_{n}\right)(t) \stackrel{n \rightarrow \infty}{\rightarrow}(\boldsymbol{j} \boldsymbol{x})(t) & \text { in } H \quad \text { for a.e. } t \in I,
\end{array}
$$


and

$$
\limsup _{n \rightarrow \infty}\left\langle\mathcal{A} \boldsymbol{x}_{n}, \boldsymbol{x}_{n}-\boldsymbol{x}\right\rangle_{\mathcal{X}} \leq 0
$$

it follows that $\langle\mathcal{A} \boldsymbol{x}, \boldsymbol{x}-\boldsymbol{y}\rangle_{\mathcal{X}} \leq \liminf _{n \rightarrow \infty}\left\langle\mathcal{A} \boldsymbol{x}_{n}, \boldsymbol{x}_{n}-\boldsymbol{y}\right\rangle_{\mathcal{X}}$ for all $\boldsymbol{y} \in \mathcal{X}$.

We will see in the proof of Theorem 4.1 that (3.2)-(3.5) are natural properties of a sequence $\left(\boldsymbol{x}_{n}\right)_{n \in \mathbb{N}} \subseteq \mathcal{X} \cap_{\boldsymbol{j}} \mathcal{Y}$ coming from an appropriate Galerkin approximation of (2.26), if $\mathcal{A}$ satisfies appropriate additional assumptions. In fact, (3.2) usually is a consequence of the coercivity of $\mathcal{A}$, (3.2) stems from the time derivative, while (3.2) and (3.5) follow directly from the Galerkin approximation.

Remark 3.6. One easily sees, that each pseudo-monotone operator $\mathcal{A}: \mathcal{X} \rightarrow \mathcal{X}^{*}$ is Bochner pseudo-monotone with $D(\mathcal{A})=\mathcal{X}$. Note that converse is not true in general. In fact, there exist Bochner pseudo-monotone operators which are not pseudomonotone. This can be seen by the following example.

Let $I=(0,2 \pi), V=W_{0, \operatorname{div}}^{1, p}(\Omega), p>3, H=L_{\operatorname{div}}^{2}(\Omega), \mathcal{X}=L^{p}(I, V)$, $\mathcal{Y}=L^{\infty}(I, H)$ and let $B: V \rightarrow V^{*}$ be the convective term, defined through $\langle B \mathbf{v}, \mathbf{w}\rangle_{V}=\int_{\Omega} \mathbf{v} \otimes \mathbf{v}: \mathbf{D w} \mathrm{d} x$ for all $\mathbf{v}, \mathbf{w} \in V$. Then, $B: V \rightarrow V^{*}$ is compact, and thus pseudo-monotone. The unsteady convective term $\mathcal{B}: \mathcal{X} \rightarrow \mathcal{X}^{*}$, given via $\langle\mathcal{B} \boldsymbol{x}, \boldsymbol{y}\rangle_{\mathcal{X}}:=\int_{I}\langle B(\boldsymbol{x}(t)), \boldsymbol{y}(t)\rangle_{V} \mathrm{~d} t$ for all $\boldsymbol{x}, \boldsymbol{y} \in \mathcal{X}$, is well-defined but neither compact nor pseudo-monotone. In fact, let $\mathbf{v}, \mathbf{w} \in V$ be fixed with $\langle B \mathbf{v}, \mathbf{w}\rangle_{V}<0$ and $\left(\boldsymbol{x}_{n}\right)_{n \in \mathbb{N}} \subseteq \mathcal{X}$, given via $\boldsymbol{x}_{n}(t)=\sin (n t) \mathbf{v}$ for every $t \in I$ and $n \in \mathbb{N}$. Then, $\boldsymbol{x}_{n} \rightarrow \mathbf{0}$ in $\mathcal{X}(n \rightarrow \infty)$ and $\lim \sup _{n \rightarrow \infty}\left\langle\mathcal{B} \boldsymbol{x}_{n}, \boldsymbol{x}_{n}-\mathbf{0}\right\rangle_{\mathcal{X}}=0$, since $\left\langle\mathcal{B} \boldsymbol{x}_{n}, \boldsymbol{x}_{n}\right\rangle_{\mathcal{X}}=0$. For $\boldsymbol{y}(\cdot):=\mathbf{w} \in \mathcal{X}$ it holds $\liminf _{n \rightarrow \infty}\left\langle\mathcal{B} \boldsymbol{x}_{n}, \boldsymbol{x}_{n}-\boldsymbol{y}\right\rangle_{\mathcal{X}}=\pi\langle B \mathbf{v}, \mathbf{w}\rangle_{V}<0=$ $\langle\mathcal{B 0}, \mathbf{0}-\boldsymbol{y}\rangle_{\mathcal{X}}$, i.e., $\mathcal{B}$ is not pseudo-monotone, and thus also not compact. We emphasize that even $\boldsymbol{x}_{n} \stackrel{*}{\rightarrow} \mathbf{0}$ in $L^{\infty}(I, V)(n \rightarrow \infty)$, but $\boldsymbol{x}_{n}(t) \rightarrow \mathbf{0}$ in $H$ for almost every $t \in I(n \rightarrow \infty)$ is not satisfied, which is the additional requirement in the Bochner pseudo-monotonicity.

On the other hand, $\mathcal{B}: \mathcal{X} \rightarrow \mathcal{X}^{*}$ is Bochner pseudo-monotone. If $\left(\boldsymbol{x}_{n}\right)_{n \in \mathbb{N}} \subseteq$ $\mathcal{X} \cap \boldsymbol{y} \mathcal{Y}$ satisfies (3.2)-(3.5), then we infer from Landes' and Mustonen's compactness principle (cf. [13, Propositon 1]) that $\boldsymbol{x}_{n} \rightarrow \boldsymbol{x}$ almost everywhere in $I \times \Omega(n \rightarrow \infty)$. As $\mathcal{X} \cap \boldsymbol{j} \hookrightarrow L^{\rho}(I \times \Omega)$, where $\rho=\frac{5}{3} p>2 p^{\prime}$, we thus gain $\boldsymbol{x}_{n} \otimes \boldsymbol{x}_{n} \rightarrow \boldsymbol{x} \otimes \boldsymbol{x}$ in $L^{p^{\prime}}(I \times \Omega)(n \rightarrow \infty)$, which in turn implies $\mathcal{B} \boldsymbol{x}_{n} \rightarrow \mathcal{B} \boldsymbol{x}$ in $\mathcal{X}^{*}$ and therefore $\langle\mathcal{B} \boldsymbol{x}, \boldsymbol{x}-\boldsymbol{y}\rangle_{\mathcal{X}} \leq \liminf _{n \rightarrow \infty}\left\langle\mathcal{B} \boldsymbol{x}_{n}, \boldsymbol{x}_{n}-\boldsymbol{y}\right\rangle \mathcal{X}$ for any $\boldsymbol{y} \in \mathcal{X}$.

The convective term is for $p>\frac{11}{5}$ well-defined as an operator $\mathcal{B}: \mathcal{X} \cap j \mathcal{Y} \rightarrow \mathcal{X}^{*}$, as can be easily checked by Hölder's inequality. Note that the above argumentation works also for $p>\frac{11}{5}$, since $\rho=\frac{5}{3} p>2 p^{\prime}$. Thus, $\mathcal{B}: D(\mathcal{A}) \subseteq \mathcal{X} \rightarrow \mathcal{X}^{*}$ with $D(\mathcal{A})=\mathcal{X} \cap{ }_{j} \mathcal{Y}$ is Bochner pseudo-monotone for $p>\frac{11}{5}$.

Pseudo-monotonicity possesses two essential properties. On the one hand, if $A$ : $X \rightarrow X^{*}$ is pseudo-monotone, $\left(x_{n}\right)_{n \in \mathbb{N}} \subseteq X$ satisfies (2.2)-(2.3) and $\left(A x_{n}\right)_{n \in \mathbb{N}} \subseteq X^{*}$ is bounded, then $A x_{n} \stackrel{n \rightarrow \infty}{\rightarrow} A x$ in $X^{*}$. On the other hand, pseudo-monotonicity is stable under summation, in the sense that the sum of two pseudo-monotone operators is 
pseudo-monotone again. We will see that Bochner pseudo-monotonicity also possesses these two essential properties.

Proposition 3.7. Let $(V, H, j)$ be a pre-evolution triple, $1<p<\infty$ and $\mathcal{A}$ : $D(\mathcal{A}) \subseteq \mathcal{X} \rightarrow \mathcal{X}^{*}$ be Bochner pseudo-monotone. Then it holds:

(i) If a sequence $\left(\boldsymbol{x}_{n}\right)_{n \in \mathbb{N}} \subseteq \mathcal{X} \cap_{\boldsymbol{j}} \mathcal{Y}$ and $\boldsymbol{x} \in \mathcal{X} \cap_{j} \mathcal{Y}$ satisfy (3.2)-(3.5), and if $\left(\mathcal{A} \boldsymbol{x}_{n}\right)_{n \in \mathbb{N}} \subseteq \mathcal{X}^{*}$ is bounded, then $\mathcal{A} \boldsymbol{x}_{n} \stackrel{n \rightarrow \infty}{\rightarrow} \mathcal{A x}$ in $\mathcal{X}^{*}$

(ii) If $\mathcal{B}: D(\mathcal{B}) \subseteq \mathcal{X} \rightarrow \mathcal{X}^{*}$ is Bochner pseudo-monotone, then $\mathcal{A}+\mathcal{B}: D(\mathcal{A}) \cap$ $D(\mathcal{B}) \rightarrow \mathcal{X}^{*}$ is Bochner pseudo-monotone.

Proof. ad (i) Since $\mathcal{X}^{*}$ is reflexive, we obtain a subsequence $\left(\mathcal{A} \boldsymbol{x}_{n}\right)_{n \in \Lambda} \subseteq \mathcal{X}^{*}$ with $\Lambda \subseteq \mathbb{N}$ and $\xi \in \mathcal{X}^{*}$ such that $\mathcal{A} \boldsymbol{x}_{n} \stackrel{n \rightarrow \infty}{\rightarrow} \xi$ in $\mathcal{X}^{*}(n \in \Lambda)$. This, the Bochner pseudo-monotonicity of $\mathcal{A}: D(\mathcal{A}) \subseteq \mathcal{X} \rightarrow \mathcal{X}^{*}$ and (3.5) imply

$$
\begin{aligned}
\langle\mathcal{A} \boldsymbol{x}, \boldsymbol{x}-\boldsymbol{y}\rangle_{\mathcal{X}} & \leq \liminf _{\substack{n \rightarrow \infty \\
n \in \Lambda}}\left\langle\mathcal{A} \boldsymbol{x}_{n}, \boldsymbol{x}_{n}-\boldsymbol{y}\right\rangle_{\mathcal{X}} \\
& \leq \limsup _{\substack{n \rightarrow \infty \\
n \in \Lambda}}\left\langle\mathcal{A} \boldsymbol{x}_{n}, \boldsymbol{x}_{n}-\boldsymbol{x}\right\rangle_{\mathcal{X}}+\limsup _{\substack{n \rightarrow \infty \\
n \in \Lambda}}\left\langle\mathcal{A} \boldsymbol{x}_{n}, \boldsymbol{x}-\boldsymbol{y}\right\rangle_{\mathcal{X}} \leq\langle\boldsymbol{\xi}, \boldsymbol{x}-\boldsymbol{y}\rangle_{\mathcal{X}}
\end{aligned}
$$

for all $\boldsymbol{y} \in \mathcal{X}$ and therefore $\mathcal{A} \boldsymbol{x}=\boldsymbol{\xi}$ in $\mathcal{X}^{*}$. As this argumentation stays valid for each subsequence of $\left(\mathcal{A} \boldsymbol{x}_{n}\right)_{n \in \mathbb{N}} \subseteq \mathcal{X}^{*}, \mathcal{A} \boldsymbol{x} \in \mathcal{X}^{*}$ is a weak accumulation point of each subsequence of $\left(\mathcal{A} \boldsymbol{x}_{n}\right)_{n \in \mathbb{N}}$. Thus, the standard convergence principle (cf. [8, Kap. I, Lemma 5.4]) yields the assertion.

ad (ii) Let $\left(\boldsymbol{x}_{n}\right)_{n \in \mathbb{N}} \subseteq \mathcal{X} \cap_{\boldsymbol{j}} \mathcal{Y}$ satisfy (3.2)-(3.2) and lim $\sup _{n \rightarrow \infty}$ $\left\langle(\mathcal{A}+\mathcal{B}) \boldsymbol{x}_{n}, \boldsymbol{x}_{n}-\boldsymbol{x}\right\rangle_{\mathcal{X}} \leq 0$. Set $a_{n}:=\left\langle\mathcal{A} \boldsymbol{x}_{n}, \boldsymbol{x}_{n}-\boldsymbol{x}\right\rangle_{\mathcal{X}}$ and $b_{n}:=\left\langle\mathcal{B} \boldsymbol{x}_{n}, \boldsymbol{x}_{n}-\right.$ $\boldsymbol{x}\rangle \mathcal{X}$ for $n \in \mathbb{N}$. Then, it holds $\limsup _{n \rightarrow \infty} a_{n} \leq 0$ and $\lim _{\sup _{n \rightarrow \infty}} b_{n} \leq 0$. In fact, suppose on the contrary, e.g., that $\lim _{\sup _{n \rightarrow \infty}} a_{n}=a>0$. Then, there exists a subsequence such that $a_{n_{k}} \rightarrow a(k \rightarrow \infty)$, and therefore $\lim _{\sup _{k \rightarrow \infty}} b_{n_{k}} \leq$ $\limsup \sup _{k \rightarrow \infty} a_{n_{k}}+b_{n_{k}}-\lim _{k \rightarrow \infty} a_{n_{k}} \leq-a<0$, i.e., a contradiction, since then the Bochner pseudo-monotonicity of $\mathcal{B}: D(\mathcal{B}) \subseteq \mathcal{X} \rightarrow \mathcal{X}^{*}$ implies $0 \leq$ $\lim \inf _{n \rightarrow \infty, n \in \Lambda} b_{n}<0$. Thus, we have $\limsup _{n \rightarrow \infty} a_{n} \leq 0$ and $\lim _{\sup _{n \rightarrow \infty}} b_{n} \leq 0$, and the Bochner pseudo-monotonicity of the operators $\mathcal{A}$ and $\mathcal{B}$ provides $\langle\mathcal{A} \boldsymbol{x}, \boldsymbol{x}-$ $\boldsymbol{y}\rangle_{\mathcal{X}} \leq \liminf _{n \rightarrow \infty}\left\langle\mathcal{A} \boldsymbol{x}_{n}, \boldsymbol{x}_{n}-\boldsymbol{y}\right\rangle_{\mathcal{X}}$ and $\langle\mathcal{B} \boldsymbol{x}, \boldsymbol{x}-\boldsymbol{y}\rangle_{\mathcal{X}} \leq \liminf _{n \rightarrow \infty}$ $\left\langle\mathcal{B} \boldsymbol{x}_{n}, \boldsymbol{x}_{n}-\boldsymbol{y}\right\rangle_{\mathcal{X}}$. Summing these inequalities yields the assertion.

Definition 3.8. (Bochner coercivity) Let $(V, H, j)$ be a pre-evolution triple and $1<$ $p<\infty$. An operator $\mathcal{A}: D(\mathcal{A}) \subseteq \mathcal{X} \rightarrow \mathcal{X}^{*}$ with $D(\mathcal{A}) \supseteq \mathcal{X} \cap j \mathcal{Y}$ is said to be

(i) Bochner coercive with respect to $f \in \mathcal{X}^{*}$ and $x_{0} \in H$ if there exists a constant $M:=M\left(f, x_{0}, \mathcal{A}\right)>0$ such that for all $x \in \mathcal{X} \cap_{j} \mathcal{Y}$ from

$$
\frac{1}{2}\|(\boldsymbol{j} \boldsymbol{x})(t)\|_{H}^{2}+\left\langle\mathcal{A} \boldsymbol{x}-\boldsymbol{f}, \boldsymbol{x} \chi_{[0, t]}\right\rangle \mathcal{X} \leq \frac{1}{2}\left\|\boldsymbol{x}_{0}\right\|_{H}^{2} \quad \text { for a.e. } t \in I
$$

it follows that $\|\boldsymbol{x}\| \boldsymbol{X}_{\cap_{j}} \mathcal{Y} \leq M$.

(ii) Bochner coercive if it is Bochner coercive with respect to $f$ and $\boldsymbol{x}_{0}$ for all $f \in \mathcal{X}^{*}$ and $\boldsymbol{x}_{0} \in H$. 
Note that Bochner coercivity, similar to semi-coercivity (cf. [18]) in conjunction with Gronwall's inequality, takes into account the information from the operator and the time derivative. In fact, Bochner coercivity is a more general property. In the context of the main theorem on pseudo-monotone perturbations of maximal monotone mapping (cf. [24, §32.4.]), which implies Theorem 1.3, Bochner coercivity is phrased in the spirit of a local coercivity ${ }^{4}$ type condition. By this we mean, if $\mathcal{A}: D(\mathcal{A}) \subseteq$ $\mathcal{X} \rightarrow \mathcal{X}^{*}$ is Bochner coercive with respect to $f \in \mathcal{X}^{*}$ and $\boldsymbol{x}_{0} \in H$, then for $\boldsymbol{x} \in \mathcal{W}$ from $\left\|\left(\boldsymbol{j}_{c} \boldsymbol{x}\right)(0)\right\|_{H} \leq\left\|\boldsymbol{x}_{0}\right\|_{H}$, i.e., $\int_{I}\left\langle\frac{d_{e} \boldsymbol{x}}{\mathrm{d} t}(t), \boldsymbol{x}(t)\right\rangle_{V \cap_{j} H} \mathrm{~d} t \geq-\frac{1}{2}\left\|\boldsymbol{x}_{0}\right\|_{H}^{2}$, and

$$
\int_{0}^{t}\left\langle\frac{d_{e} \boldsymbol{x}}{\mathrm{d} t}(t), \boldsymbol{x}(t)\right\rangle_{V \cap_{j} H} \mathrm{~d} t+\left\langle\mathcal{A} \boldsymbol{x}, \boldsymbol{x} \chi_{[0, t]}\right\rangle \mathcal{X} \leq\left\langle\boldsymbol{f}, \boldsymbol{x} \chi_{[0, t]}\right\rangle \mathcal{X} \quad \text { for a.e. } t \in I,
$$

it follows $\|\boldsymbol{x}\| \boldsymbol{\mathcal { X }} \cap_{j} \mathcal{Y} \leq M$, since (3.10) is just (3.9). In other words, if the image of $\boldsymbol{x} \in \mathcal{W}$ with respect to $\frac{d_{e}}{\mathrm{~d} t}$ and $\mathcal{A}$ is bounded by the data $\boldsymbol{x}_{0}, \boldsymbol{f}$ in this weak sense, then $\boldsymbol{x}$ is contained in a fixed ball in $\mathcal{X} \cap \boldsymbol{Y}$. We chose (3.9) instead of (3.10) in Definition 3.8, since $\boldsymbol{x} \in \mathcal{X} \cap \boldsymbol{j}$ is not admissible in (3.10).

Apart from that, there is a relation between Bochner coercivity and coercivity in the sense of Definition 2.1. In fact, in the case of bounded operators $\mathcal{A}: D(\mathcal{A}) \subseteq \mathcal{X} \rightarrow$ $\mathcal{X}^{*}$, Bochner coercivity extends the standard concept of coercivity.

Lemma 3.11. Let $(V, H, j)$ be a pre-evolution triple and $1<p<\infty$. If the operator $\mathcal{A}: D(\mathcal{A}) \subseteq \mathcal{X} \rightarrow \mathcal{X}^{*}$ with $D(\mathcal{A})=\mathcal{X} \cap{ }_{j} \mathcal{Y}$ is coercive and bounded, then $\mathcal{A}$ is Bochner coercive.

Proof. It suffices to show that $\mathcal{A}: D(\mathcal{A}) \subseteq \mathcal{X} \rightarrow \mathcal{X}^{*}$ is Bochner coercive with respect to $\mathbf{0} \in \mathcal{X}^{*}$ and $\boldsymbol{x}_{0} \in H$. For $\boldsymbol{f} \in \mathcal{X}^{*} \backslash\{\mathbf{0}\}$, we consider the shifted operator $\widehat{\mathcal{A}}:=\mathcal{A}-f: D(\mathcal{A}) \subset \mathcal{X} \rightarrow \mathcal{X}^{*}$ which is still coercive and bounded. Therefore, $\widehat{\mathcal{A}}$ is Bochner coercive with respect to $\mathbf{0} \in \mathcal{X}^{*}$ and $\boldsymbol{x}_{0} \in H$, and we conclude that $\mathcal{A}$ is Bochner coercive. To show that $\mathcal{A}: \mathcal{X} \cap_{j} \mathcal{Y} \rightarrow \mathcal{X}^{*}$ is Bochner coercive with respect to $\mathbf{0}$ and $\boldsymbol{x}_{0}$, we assume that $\boldsymbol{x} \in \mathcal{X} \cap \boldsymbol{j}$ satisfies for almost every $t \in I$

$$
\frac{1}{2}\|(\boldsymbol{j} \boldsymbol{x})(t)\|_{H}^{2}+\left\langle\mathcal{A} \boldsymbol{x}, \boldsymbol{x} \chi_{[0, t]}\right\rangle \mathcal{X} \leq \frac{1}{2}\left\|\boldsymbol{x}_{0}\right\|_{H}^{2} .
$$

Since $\mathcal{A}: D(\mathcal{A}) \subset \mathcal{X} \rightarrow \mathcal{X}^{*}$ is coercive there exists a constant $R:=R(\mathcal{A})>0$ such that $\langle\mathcal{A} w, w\rangle_{\mathcal{X}} \geq\|\boldsymbol{w}\|_{\mathcal{X}}$ for all $\boldsymbol{w} \in D(\mathcal{A})=\mathcal{X} \cap_{j} \mathcal{Y}$ such that $\|\boldsymbol{w}\|_{\mathcal{X}} \geq R$. Next, we define $M_{0}:=\max \left\{R, \frac{1}{2}\left\|\boldsymbol{x}_{0}\right\|_{H}^{2}\right\}>0$ and suppose that $\|\boldsymbol{x}\|_{\mathcal{X}}>M_{0} \geq R$. Therefore, using the coercivity and (3.12), we conclude $M_{0}<\|\boldsymbol{x}\|_{\mathcal{X}} \leq\langle\mathcal{A} \boldsymbol{x}, \boldsymbol{x}\rangle_{\mathcal{X}} \leq$ $\frac{1}{2}\left\|\boldsymbol{x}_{0}\right\|_{H}^{2} \leq M_{0}$, which is a contradiction. Thus, $\|\boldsymbol{x}\|_{\mathcal{X}} \leq M_{0}$ has to be valid. As $\mathcal{A}: D(\mathcal{A}) \subset \mathcal{X} \rightarrow \mathcal{X}^{*}$ is bounded there exists a constant $\Lambda:=\Lambda\left(M_{0}\right)>0$ such

${ }^{4} A: D(A) \subseteq V \rightarrow V^{*}$ is said to be coercive (cf. [24, \$32.4.]) with respect to $f \in V^{*}$, if $D(A)$ is unbounded and there exists a constant $R>0$, such that for $v \in V$ from $\langle A v, v\rangle_{V} \leq\langle f, v\rangle_{V}$ it follows $\|v\|_{V} \leq R$, i.e., all elements whose images with respect to $A$ do not grow beyond the data $f$ in this weak sense are contained in a fixed ball in $V$. 
that $\|\mathcal{A} w\|_{\mathcal{X}^{*}} \leq \Lambda$ for all $\boldsymbol{w} \in \mathcal{X} \cap_{j} \mathcal{Y}$ with $\|\boldsymbol{w}\|_{\mathcal{X}} \leq M_{0}$. This and (3.12) imply $\|\boldsymbol{j} \boldsymbol{x}\|_{\mathcal{Y}}^{2} \leq\left\|\boldsymbol{x}_{0}\right\|_{H}^{2}+2 \Lambda M_{0}$, which yields $\|\boldsymbol{x}\| \boldsymbol{X}_{\cap_{j}} \mathcal{Y} \leq M_{0}+\left(\left\|\boldsymbol{x}_{0}\right\|_{H}^{2}+2 \Lambda M_{0}\right)^{1 / 2}=$ : $M$.

The following proposition provides sufficient conditions on a time-dependent family of operators such that the induced operator is bounded, Bochner pseudo-monotone and Bochner coercive.

Proposition 3.13. Let $(V, H, j)$ be a pre-evolution triple and $1<p<\infty$. Furthermore, let $A(t): V \cap_{j} H \rightarrow\left(V \cap_{j} H\right)^{*}, t \in I$, be a family of operators satisfying (C.1) and (C.2). Then it holds:

(i) If in addition $\{A(t)\}_{t \in I}$ satisfies (C.3), where

(C.3) For some nonnegative functions $\alpha, \gamma \in L^{p^{\prime}}(I), \beta \in L^{\infty}(I)$ and a non-decreasing function $\mathscr{B}: \mathbb{R}_{\geq 0} \rightarrow \mathbb{R}_{\geq 0}$ holds

$$
\|A(t) v\|_{\left(V \cap_{j} H\right)^{*}} \leq \mathscr{B}\left(\|j v\|_{H}\right)\left(\alpha(t)+\beta(t)\|v\|_{V}^{p-1}\right)+\gamma(t)
$$

for almost every $t \in I$ and all $v \in V \cap_{j} H$.

then the induced operator $\mathcal{A}:=R \boldsymbol{A}: D(\mathcal{A}) \subseteq \mathcal{X} \rightarrow \mathcal{X}^{*}$ with $D(\mathcal{A})=$ $\mathcal{X} \cap_{j} \mathcal{Y}$, given via (2.24), is well-defined. Moreover, $\mathcal{A}$ maps bounded sets in $\mathcal{X} \cap j \mathcal{Y}$ into bounded sets in $\mathcal{X}^{*}$, i.e., $\mathcal{A}$ viewed as an operator from $\mathcal{X} \cap \boldsymbol{j}$ into $\mathcal{X}^{*}$ is bounded.

(ii) If in addition $\{A(t)\}_{t \in I}$ satisfies (C.3), (C.4) and (C.5), where

(C.4) $A(t): V \cap_{j} H \rightarrow\left(V \cap_{j} H\right)^{*}$ is pseudo-monotone for almost every $t \in I$.

(C.5) For some constant $c_{0}>0$, nonnegative functions $c_{1}, c_{2} \in L^{1}(I)$ and a non-decreasing function $\mathscr{C}: \mathbb{R}_{\geq 0} \rightarrow \mathbb{R}_{\geq 0}$ holds

$$
\langle A(t) v, v\rangle_{V \cap_{j} H} \geq c_{0}\|v\|_{V}^{p}-c_{1}(t) \mathscr{C}\left(\|j v\|_{H}\right)-c_{2}(t)
$$

for almost every $t \in I$ and all $v \in V \cap_{j} H$.

then $\mathcal{A}: D(\mathcal{A}) \subseteq \mathcal{X} \rightarrow \mathcal{X}^{*}$ with $D(\mathcal{A})=\mathcal{X} \cap{ }_{j} \mathcal{Y}$ is Bochner pseudomonotone.

(iii) If in addition $\{A(t)\}_{t \in I}$ satisfies (C.3), (C.4) and (C.5) with $\mathscr{C}(s)=s^{2}$, then $\mathcal{A}: D(\mathcal{A}) \subseteq \mathcal{X} \rightarrow \mathcal{X}^{*}$ with $D(\mathcal{A})=\mathcal{X} \cap j \mathcal{Y}$ is Bochner coercive.

We emphasize that in applications the conditions (C.1), (C.2), (C.4) and (C.5) are usually directly deducible from the corresponding steady problem given trough the operator $A(t): V \cap_{j} H \rightarrow\left(V \cap_{j} H\right)^{*}$ for fixed $t \in I$. Only the verification of (C.3) sometimes causes some moderate effort. These circumstances are illustrated in the Examples 5.1 and 5.2.

Proof. ad (i) 1. Well-definedness: Let $\boldsymbol{x} \in \mathcal{X} \cap \boldsymbol{j} \mathcal{Y}$. According to Lemma 2.18 the function $(t \mapsto \boldsymbol{A x}(t)): I \rightarrow\left(V \cap_{j} H\right)^{*}$ is Bochner measurable. In consequence, the 
function $\left(t \mapsto\langle(\boldsymbol{A} \boldsymbol{x})(t), \boldsymbol{y}(t)\rangle_{V \cap_{j} H}\right): I \rightarrow \mathbb{R}$ is for every $\boldsymbol{y} \in \mathcal{X}$ Lebesgue measurable. Therefore, we can inspect it for integrability. In doing so, applying Hölder's inequality and using (C.3), we obtain

$$
\begin{aligned}
& \int_{I}\left|\langle(\boldsymbol{A} \boldsymbol{x})(s), \boldsymbol{y}(s)\rangle_{V \cap_{j} H}\right| \mathrm{d} s \\
& \quad \leq\left(\int_{I}\|A(s)(\boldsymbol{x}(s))\|_{\left(V \cap_{j} H\right)^{*}}^{p^{\prime}} \mathrm{d} s\right)^{\frac{1}{p^{\prime}}}\left(\int_{I}\|\boldsymbol{y}(s)\|_{V \cap_{j} H}^{p} \mathrm{~d} s\right)^{\frac{1}{p}} \\
& \quad \leq\left(\int_{I}\left(\mathscr{B}\left(\|j(\boldsymbol{x}(s))\|_{H}\right)\left(\alpha(s)+\beta(s)\|\boldsymbol{x}(s)\|_{V}^{p-1}\right)+\gamma(s)\right)^{p^{\prime}} d s\right)^{\frac{1}{p^{\prime}}}\|\boldsymbol{y}\| \mathcal{X} \\
& \quad \leq \mathscr{B}(\|\boldsymbol{j} \boldsymbol{x}\| \mathcal{Y})\left(\left(\|\alpha\|_{L^{p^{\prime}(I)}}+\|\beta\|_{L^{\infty}(I)}\|\boldsymbol{x}\|_{\mathcal{X}}^{p-1}\right)+\|\gamma\|_{L^{p^{\prime}(I)}}\right)\|\boldsymbol{y}\| \boldsymbol{\mathcal { X }} .
\end{aligned}
$$

As a result, we obtain $\mathcal{A} \boldsymbol{x} \in \mathcal{X}^{*}$.

2. Boundedness: The boundedness of $\mathcal{A}: D(\mathcal{A}) \subseteq \mathcal{X} \rightarrow \mathcal{X}^{*}$ with $D(\mathcal{A})=$ $\mathcal{X} \cap j \mathcal{Y}$ also follows from the estimate (3.14).

ad (ii) The presented proof is a generalization of [1, Lemma 4.2] and uses ideas from $[10,11,13,20]$. Our approach completely avoids additional technical assumptions on the spaces, as e.g., the existence of certain projections, which were present in previous investigations. We proceed in four steps:

1. Collecting information: Let $\left(x_{n}\right)_{n \in \mathbb{N}} \subseteq \mathcal{X} \cap_{j} \mathcal{Y}$ be a sequence satisfying (3.2)(3.5). Thus, $\left(\boldsymbol{x}_{n}\right)_{n \in \mathbb{N}}$ is bounded in $\mathcal{X} \cap \boldsymbol{j} \mathcal{Y}$, and due to (i) the sequence $\left(\mathcal{A} \boldsymbol{x}_{n}\right)_{n \in \mathbb{N}}$ is bounded in $\mathcal{X}^{*}$. From the reflexivity of $\mathcal{X}^{*}$ we obtain a subsequence $\left(\boldsymbol{x}_{n}\right)_{n \in \Lambda}$ with $\Lambda \subseteq$ $\mathbb{N}$ and $\boldsymbol{\xi} \in \mathcal{X}^{*}$ such that $\mathcal{A} \boldsymbol{x}_{n} \stackrel{n \rightarrow \infty}{\rightarrow} \xi$ in $\mathcal{X}^{*}(n \in \Lambda)$ and $\lim _{n \rightarrow \infty}\left\langle\mathcal{A} \boldsymbol{x}_{n}, \boldsymbol{x}_{n}\right\rangle \mathcal{X}=$ $\liminf _{n \rightarrow \infty}\left\langle\mathcal{A} \boldsymbol{x}_{n}, \boldsymbol{x}_{n}\right\rangle \mathcal{X}$. Thus, we have for all $\boldsymbol{y} \in \mathcal{X}$

$$
\lim _{\substack{n \rightarrow \infty \\ n \in \Lambda}}\left\langle\mathcal{A} \boldsymbol{x}_{n}, \boldsymbol{x}_{n}-\boldsymbol{y}\right\rangle_{\mathcal{X}} \leq \liminf _{n \rightarrow \infty}\left\langle\mathcal{A} \boldsymbol{x}_{n}, \boldsymbol{x}_{n}-\boldsymbol{y}\right\rangle_{\mathcal{X}}
$$

Due to (3.2) there exists a subset $E \subseteq I$ such that $I \backslash E$ is a null set and for all $t \in E$

$$
\left(\boldsymbol{j} \boldsymbol{x}_{n}\right)(t) \stackrel{n \rightarrow \infty}{\rightarrow}(\boldsymbol{j} \boldsymbol{x})(t) \quad \text { in } H .
$$

In addition, using (C.3) and (C.5) we get

$$
\begin{aligned}
\langle A(t) & \left.\left(\boldsymbol{x}_{n}(t)\right), \boldsymbol{x}_{n}(t)-\boldsymbol{x}(t)\right\rangle_{V \cap_{j} H} \\
\geq & c_{0}\left\|\boldsymbol{x}_{n}(t)\right\|_{V}^{p}-c_{1}(t) \mathscr{C}\left(\left\|j\left(\boldsymbol{x}_{n}(t)\right)\right\|_{H}\right)-c_{2}(t)-\left\langle A(t)\left(\boldsymbol{x}_{n}(t)\right), \boldsymbol{x}(t)\right\rangle_{V \cap_{j} H} \\
\geq & c_{0}\left\|\boldsymbol{x}_{n}(t)\right\|_{V}^{p}-c_{1}(t) \mathscr{C}\left(\left\|j\left(\boldsymbol{x}_{n}(t)\right)\right\|_{H}\right)-c_{2}(t) \\
& -\left(\mathscr{B}\left(\left\|j\left(\boldsymbol{x}_{n}(t)\right)\right\|_{H}\right)\left(\alpha(t)+\beta(t)\left\|\boldsymbol{x}_{n}(t)\right\|_{V}^{p-1}\right)+\gamma(t)\right)\|\boldsymbol{x}(t)\|_{V \cap_{j} H}
\end{aligned}
$$

for almost every $t \in I$. From $\left\|\boldsymbol{j} \boldsymbol{x}_{n}\right\| \mathcal{Y} \leq K$ for some constant $K>0$, which follows from (3.2), and the $\varepsilon$-Young inequality with $k:=k(\varepsilon, p):=\left(p^{\prime} \varepsilon\right)^{1-p} / p$ and $\varepsilon:=\left(\mathscr{B}(K)\|\beta\|_{L^{\infty}(I)}\right)^{-p^{\prime}} c_{0} / 2$ we further obtain for all $n \in \Lambda$ and for almost every $t \in I$ 


$$
\left\langle A(t)\left(\boldsymbol{x}_{n}(t)\right), \boldsymbol{x}_{n}(t)-\boldsymbol{x}(t)\right\rangle_{V \cap_{j} H} \geq \frac{c_{0}}{2}\left\|\boldsymbol{x}_{n}(t)\right\|_{V}^{p}-\mu_{\boldsymbol{x}}(t), \quad(*)_{n, t}
$$

where $\mu_{\boldsymbol{x}}(t):=c_{1}(t) \mathscr{C}(K)+c_{2}(t)+k\|\boldsymbol{x}(t)\|_{V \cap_{j} H}^{p}+(\mathscr{B}(K) \alpha(t)+\gamma(t))\|\boldsymbol{x}(t)\|_{V \cap_{j} H}$ $\in L^{1}(I)$. Next, we define

$$
\begin{gathered}
\mathcal{S}:=\left\{t \in E \mid A(t): V \cap_{j} H \rightarrow\left(V \cap_{j} H\right)^{*}\right. \text { is pseudo-monotone, } \\
\left.\left|\mu_{x}(t)\right|<\infty \text { and }(*)_{n, t} \text { holds for all } n \in \Lambda\right\}
\end{gathered}
$$

Apparently, $I \backslash \mathcal{S}$ is a null set.

2. Intermediate objective: Our next objective is to verify for all $t \in \mathcal{S}$

$$
\liminf _{\substack{n \rightarrow \infty \\ n \in \Lambda}}\left\langle A(t)\left(\boldsymbol{x}_{n}(t)\right), \boldsymbol{x}_{n}(t)-\boldsymbol{x}(t)\right\rangle_{V \cap_{j} H} \geq 0 .
$$

To this end, let us fix an arbitrary $t \in \mathcal{S}$ and define

$$
\Lambda_{t}:=\left\{n \in \Lambda \mid\left\langle A(t)\left(\boldsymbol{x}_{n}(t)\right), \boldsymbol{x}_{n}(t)-\boldsymbol{x}(t)\right\rangle_{V \cap_{j} H}<0\right\}
$$

We assume without loss of generality that $\Lambda_{t}$ is not finite. Otherwise, $(* *)_{t}$ would already hold true for this specific $t \in \mathcal{S}$ and nothing would be left to do. But if $\Lambda_{t}$ is not finite, then

$$
\limsup _{\substack{n \rightarrow \infty \\ n \in \Lambda_{t}}}\left\langle A(t)\left(\boldsymbol{x}_{n}(t)\right), \boldsymbol{x}_{n}(t)-\boldsymbol{x}(t)\right\rangle_{V \cap_{j} H} \leq 0 .
$$

From (3.17) and $(*)_{n, t}$ follows for all $n \in \Lambda_{t}$

$$
\frac{c_{0}}{2}\left\|\boldsymbol{x}_{n}(t)\right\|_{V}^{p} \leq\left\langle A(t)\left(\boldsymbol{x}_{n}(t)\right), \boldsymbol{x}_{n}(t)-\boldsymbol{x}(t)\right\rangle_{V \cap_{j} H}+\left|\mu_{\boldsymbol{x}}(t)\right|<\left|\mu_{\boldsymbol{x}}(t)\right|<\infty .
$$

Thanks to (3.16) and (3.18), Proposition 2.9 (iv) yields that

$$
\boldsymbol{x}_{n}(t) \stackrel{n \rightarrow \infty}{\rightarrow} \boldsymbol{x}(t) \quad \text { in } V \cap_{j} H\left(n \in \Lambda_{t}\right)
$$

The pseudo-monotonicity of $A(t): V \cap_{j} H \rightarrow\left(V \cap_{j} H\right)^{*}$ finally guarantees

$$
\liminf _{\substack{n \rightarrow \infty \\ n \in \Lambda_{t}}}\left\langle A(t)\left(\boldsymbol{x}_{n}(t)\right), \boldsymbol{x}_{n}(t)-\boldsymbol{x}(t)\right\rangle_{V \cap_{j} H} \geq 0 .
$$

Due to $\left\langle A(t)\left(\boldsymbol{x}_{n}(t)\right), \boldsymbol{x}_{n}(t)-\boldsymbol{x}(t)\right\rangle_{V \cap_{j} H} \geq 0$ for all $n \in \Lambda \backslash \Lambda_{t},(* *)_{t}$ holds for all $t \in \mathcal{S}$.

3. Switching to the image space level: In this passage we verify the existence of a subsequence $\left(\boldsymbol{x}_{n}\right)_{n \in \Lambda_{0}} \subseteq \mathcal{X} \cap_{j} \mathcal{Y}$ with $\Lambda_{0} \subseteq \Lambda$ such that for almost every $t \in I$

$$
\begin{array}{r}
\boldsymbol{x}_{n}(t) \stackrel{n \rightarrow \infty}{\rightarrow} \boldsymbol{x}(t) \quad \text { in } V \cap_{j} H\left(n \in \Lambda_{0}\right), \\
\limsup _{\substack{n \rightarrow \infty \\
n \in \Lambda_{0}}}\left\langle A(t)\left(\boldsymbol{x}_{n}(t)\right), \boldsymbol{x}_{n}(t)-\boldsymbol{x}(t)\right\rangle_{V \cap_{j} H} \leq 0 .
\end{array}
$$


As a consequence, we are in a position to exploit the almost everywhere pseudomonotonicity of the operator family. Thanks to $\left\langle A(t)\left(\boldsymbol{x}_{n}(t)\right), \boldsymbol{x}_{n}(t)-\boldsymbol{x}(t)\right\rangle_{V \cap_{j} H} \geq$ $-\mu_{\boldsymbol{x}}(t)$ for all $t \in \mathcal{S}$ and $n \in \Lambda$, Fatou's lemma (cf. [18, Theorem 1.18]) is applicable. It yields, also using $(* *)_{t}$ and (3.5)

$$
\begin{aligned}
0 & \leq \int_{I} \liminf _{\substack{n \rightarrow \infty \\
n \in \Lambda}}\left\langle A(s)\left(\boldsymbol{x}_{n}(s)\right), \boldsymbol{x}_{n}(s)-\boldsymbol{x}(s)\right\rangle_{V \cap_{j} H} \mathrm{~d} s \\
& \leq \liminf _{\substack{n \rightarrow \infty \\
n \in \Lambda}} \int_{I}\left\langle A(s)\left(\boldsymbol{x}_{n}(s)\right), \boldsymbol{x}_{n}(s)-\boldsymbol{x}(s)\right\rangle_{V \cap_{j} H} \mathrm{~d} s \leq \limsup _{n \rightarrow \infty}\left\langle\mathcal{A} \boldsymbol{x}_{n}, \boldsymbol{x}_{n}-\boldsymbol{x}\right\rangle \mathcal{X} \leq 0 .
\end{aligned}
$$

Let us define $h_{n}(t):=\left\langle A(t)\left(\boldsymbol{x}_{n}(t)\right), \boldsymbol{x}_{n}(t)-\boldsymbol{x}(t)\right\rangle_{V \cap_{j} H}$. Then $(* *)_{t}$ and (3.20) read:

$$
\begin{aligned}
& \liminf _{\substack{n \rightarrow \infty \\
n \in \Lambda}} h_{n}(t) \geq 0 \quad \text { for all } t \in \mathcal{S} . \\
& \lim _{\substack{n \rightarrow \infty \\
n \in \Lambda}} \int_{I} h_{n}(s) \mathrm{d} s=0 .
\end{aligned}
$$

As $s \mapsto s^{-}:=\min \{0, s\}$ is continuous and non-decreasing we deduce from (3.21) that

$$
0 \geq \limsup _{\substack{n \rightarrow \infty \\ n \in \Lambda}} h_{n}(t)^{-} \geq \liminf _{\substack{n \rightarrow \infty \\ n \in \Lambda}} h_{n}(t)^{-} \geq \min \left\{0, \liminf _{\substack{n \rightarrow \infty \\ n \in \Lambda}} h_{n}(t)\right\}=0,
$$

i.e., $h_{n}(t)^{-} \stackrel{n \rightarrow \infty}{\rightarrow} 0(n \in \Lambda)$ for all $t \in \mathcal{S}$. Since $0 \geq h_{n}(t)^{-} \geq-\mu_{x}(t)$ for all $t \in \mathcal{S}$ and $n \in \Lambda$, Vitali's theorem yields $h_{n}^{-} \stackrel{n \rightarrow \infty}{\rightarrow} 0$ in $L^{1}(I)$. From the latter, $\left|h_{n}\right|=h_{n}-2 h_{n}^{-}$and (3.22), we conclude that $h_{n} \stackrel{n \rightarrow \infty}{\rightarrow} 0$ in $L^{1}(I)$. Thus, there exists a subsequence $\left(\boldsymbol{x}_{n}\right)_{n \in \Lambda_{0}}$ with $\Lambda_{0} \subseteq \Lambda$ and a subset $F \subseteq I$ such that $I \backslash F$ is a null set and for all $t \in F$

$$
\lim _{\substack{n \rightarrow \infty \\ n \in \Lambda_{0}}}\left\langle A(t)\left(\boldsymbol{x}_{n}(t)\right), \boldsymbol{x}_{n}(t)-\boldsymbol{x}(t)\right\rangle_{V \cap_{j} H}=0 .
$$

Consequently, we have for all $t \in \mathcal{S} \cap F$

$\limsup _{\substack{n \rightarrow \infty \\ n \in \Lambda_{0}}} \frac{c_{0}}{2}\left\|x_{n}(t)\right\|_{V}^{p} \leq \limsup _{\substack{n \rightarrow \infty \\ n \in \Lambda_{0}}}\left\langle A(t)\left(x_{n}(t)\right), \boldsymbol{x}_{n}(t)-\boldsymbol{x}(t)\right\rangle_{V \cap_{j} H}+\left|\mu_{\boldsymbol{x}}(t)\right|=\left|\mu_{\boldsymbol{x}}(t)\right|<\infty$.

Thus, due to (3.16), Proposition 2.9 (iv) yields

$$
\boldsymbol{x}_{n}(t) \stackrel{n \rightarrow \infty}{\rightarrow} \boldsymbol{x}(t) \quad \text { in } V \cap_{j} H\left(n \in \Lambda_{0}\right)
$$

for all $t \in \mathcal{S} \cap F$. The relations (3.23) and (3.24) imply (3.19).

4. Switching to the Bochner-Lebesgue level: In view of the pseudo-monotonicity of the operators $A(t): V \cap_{j} H \rightarrow\left(V \cap_{j} H\right)^{*}$ for all $t \in \mathcal{S} \cap F$ we deduce from (3.19) that 


$$
\langle A(t)(\boldsymbol{x}(t)), \boldsymbol{x}(t)-\boldsymbol{y}(t)\rangle_{V \cap_{j} H} \leq \liminf _{\substack{n \rightarrow \infty \\ n \in \Lambda_{0}}}\left\langle A(t)\left(\boldsymbol{x}_{n}(t)\right), \boldsymbol{x}_{n}(t)-\boldsymbol{y}(t)\right\rangle_{V \cap_{j} H}
$$

almost every $t \in I$ and all $\boldsymbol{y} \in \mathcal{X}$. As in step $\mathbf{1}$ we verify that there exists $\mu_{\boldsymbol{y}} \in L^{1}(I)$ such that

$$
\left\langle A(t)\left(\boldsymbol{x}_{n}(t)\right), \boldsymbol{x}_{n}(t)-\boldsymbol{y}(t)\right\rangle_{V \cap_{j} H} \geq \frac{c_{0}}{2}\left\|\boldsymbol{x}_{n}(t)\right\|_{V}^{p}-\mu_{\boldsymbol{y}}(t)
$$

for almost every $t \in I$ and all $n \in \Lambda_{0}$. Thus, we can apply Fatou's lemma once more, exploit (3.15) and deduce further that

$$
\begin{aligned}
\langle\mathcal{A} \boldsymbol{x}, \boldsymbol{x}-\boldsymbol{y}\rangle_{\mathcal{X}} & \leq \int_{I} \liminf _{\substack{n \rightarrow \infty \\
n \in \Lambda_{0}}}\left\langle A(s)\left(\boldsymbol{x}_{n}(s)\right), \boldsymbol{x}_{n}(s)-\boldsymbol{y}(s)\right\rangle_{V \cap_{j} H} \mathrm{~d} s \\
& \leq \liminf _{\substack{n \rightarrow \infty \\
n \in \Lambda_{0}}} \int_{I}\left\langle A(s)\left(\boldsymbol{x}_{n}(s)\right), \boldsymbol{x}_{n}(s)-\boldsymbol{y}(s)\right\rangle_{V \cap_{j} H} \mathrm{~d} s \\
& =\lim _{\substack{n \rightarrow \infty \\
n \in \Lambda}}\left\langle\mathcal{A} \boldsymbol{x}_{n}, \boldsymbol{x}_{n}-\boldsymbol{y}\right\rangle_{\mathcal{X}} \\
& \leq \liminf _{n \rightarrow \infty}\left\langle\mathcal{A} \boldsymbol{x}_{n}, \boldsymbol{x}_{n}-\boldsymbol{y}\right\rangle_{\mathcal{X}}
\end{aligned}
$$

for all $\boldsymbol{y} \in \mathcal{X}$, i.e., $\mathcal{A}: D(\mathcal{A}) \subseteq \mathcal{X} \rightarrow \mathcal{X}^{*}$ with $D(\mathcal{A})=\mathcal{X} \cap_{j} \mathcal{Y}$ is Bochner pseudo-monotone.

ad (iii) As in the proof of Lemma 3.11 it suffices to show that $\mathcal{A}: D(\mathcal{A}) \subseteq \mathcal{X} \rightarrow$ $\mathcal{X}^{*}$ with $D(\mathcal{A})=\mathcal{X} \cap j \mathcal{Y}$ is Bochner coercive with respect to the origin $\mathbf{0} \in \mathcal{X}^{*}$ and $\boldsymbol{x}_{0} \in H$. To show that $\mathcal{A}: D(\mathcal{A}) \subseteq \mathcal{X} \rightarrow \mathcal{X}^{*}$ with $D(\mathcal{A})=\mathcal{X} \cap_{j} \mathcal{Y}$ is Bochner coercive with respect to $\mathbf{0}$ and $\boldsymbol{x}_{0}$, we assume that $\boldsymbol{x} \in \mathcal{X} \cap \boldsymbol{\mathcal { Y }}$ satisfies for almost every $t \in I$

$$
\frac{1}{2}\|(\boldsymbol{j} \boldsymbol{x})(t)\|_{H}^{2}+\int_{0}^{t}\langle A(s)(\boldsymbol{x}(s)), \boldsymbol{x}(s)\rangle_{V \cap_{j} H} \mathrm{~d} s \leq \frac{1}{2}\left\|\boldsymbol{x}_{0}\right\|_{H}^{2} .
$$

Using (C.5) with $\mathscr{C}(s)=s^{2}$ in (3.25) we get for almost every $t \in I$

$$
\frac{1}{2}\|(\boldsymbol{j} \boldsymbol{x})(t)\|_{H}^{2}+c_{0} \int_{0}^{t}\|\boldsymbol{x}(s)\|_{V}^{p} \mathrm{~d} s \leq \frac{1}{2}\left\|\boldsymbol{x}_{0}\right\|_{H}^{2}+\left\|c_{2}\right\|_{L^{1}(I)}+\int_{0}^{t} \mid c_{1}(s)\|\|(\boldsymbol{j} \boldsymbol{x})(s) \|_{H}^{2} \mathrm{~d} s .
$$

Gronwall's inequality (cf. [3, Lemma II.4.10]) applied on (3.26) yields

$$
\|\boldsymbol{j} \boldsymbol{x}\|_{\mathcal{Y}}^{2} \leq\left(\left\|\boldsymbol{x}_{0}\right\|_{H}^{2}+2\left\|c_{2}\right\|_{L^{1}(I)}\right) \exp \left(2\left\|c_{1}\right\|_{L^{1}(I)}\right)=: K_{0} .
$$

From (3.26) and (3.27) we further deduce that

$$
c_{0} \int_{0}^{t}\|\boldsymbol{x}(s)\|_{V}^{p} \mathrm{~d} s \leq \frac{1}{2}\left\|\boldsymbol{x}_{0}\right\|_{H}^{2}+\left\|c_{2}\right\|_{L^{1}(I)}+K_{0}\left\|c_{1}\right\|_{L^{1}(I)}=: K_{1}
$$

for all $t \in \bar{I}$. (3.27) together with (3.28) reads $\|x\|_{L^{p}(I, V) \cap_{j}} \mathcal{Y} \leq\left(K_{1} / c_{0}\right)^{\frac{1}{p}}+K_{0}^{\frac{1}{2}}$. Due

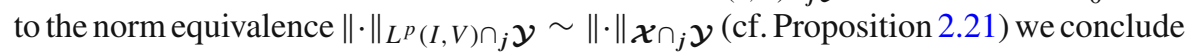
the Bochner coercivity with respect to $0 \in \mathcal{X}^{*}$ and $\boldsymbol{x}_{0} \in H$ of $\mathcal{A}: D(\mathcal{A}) \subseteq \mathcal{X} \rightarrow \mathcal{X}^{*}$ with $D(\mathcal{A})=\mathcal{X} \cap j \mathcal{Y}$. 


\section{Existence theorem}

Theorem 4.1. (Main theorem) Let $(V, H, j)$ be a pre-evolution triple, $1<p<\infty$ and $A(t): V \cap_{j} H \rightarrow(V \cap H)^{*}, t \in I$, a family of operators such that $(C .1)-(C .3)$ are fulfilled and that the induced operator $\mathcal{A}: D(\mathcal{A}) \subseteq \mathcal{X} \rightarrow \mathcal{X}^{*}$ with $D(\mathcal{A})=\mathcal{X} \cap_{j} \mathcal{Y}$, given via (2.24), is Bochner pseudo-monotone and Bochner coercive with respect to $\boldsymbol{f} \in L^{p^{\prime}}\left(I,\left(V \cap_{j} H\right)^{*}\right)$ and $\boldsymbol{y}_{0} \in H$. Then, exists a solution $\boldsymbol{y} \in \mathcal{W}$ of the evolution equation (2.26), i.e.,

$$
\begin{aligned}
& \int_{I}\left\langle\frac{d_{e} \boldsymbol{y}}{d t}(t), \boldsymbol{\varphi}(t)\right\rangle_{V \cap_{j} H} d t+\int_{I}\langle A(t)(\boldsymbol{y}(t)), \boldsymbol{\varphi}(t)\rangle_{V \cap_{j} H} d t=\int_{I}\langle\boldsymbol{f}(t), \boldsymbol{\varphi}(t)\rangle_{V \cap_{j} H} d t \quad \forall \boldsymbol{\varphi} \in \mathcal{X}, \\
& \left(\boldsymbol{j}_{c} \boldsymbol{y}\right)(0)=\boldsymbol{y}_{0} \quad \text { in } H .
\end{aligned}
$$

From Lemma 3.13 we immediately obtain the following more applicable version of Theorem 4.1.

Corollary 4.2. Let $(V, H, j)$ be an pre-evolution triple, $1<p<\infty$ and $A(t)$ : $V \cap_{j} H \rightarrow(V \cap H)^{*}, t \in I$, a family of operators such that (C.1)-(C.5) are fulfilled with $\mathscr{C}(s)=s^{2}$ in (C.5). Then, for arbitrary $\boldsymbol{y}_{0} \in H$ and $f \in L^{p^{\prime}}\left(I,\left(V \cap \cap_{j} H\right)^{*}\right)$ there exists a solution $\boldsymbol{y} \in \mathcal{W}$ of the evolution equation (2.26).

Remark 4.3. If $(V, H, j)$ is an evolution triple, the assertions of Theorem 4.1 and Corollary 4.2 remain true as $(V, H, j)$ is a pre-evolution triple as well. In addition, one can replace $V \cap_{j} H$ by $V$ in Theorem 4.1 and Corollary 4.2 as $V=V \cap_{j} H$ with norm equivalence.

\section{Proof (of Theorem 4.1)}

0. Reduction of assumptions: As in the proofs of Lemma 3.11 and Proposition 3.13 (i) it suffices anew to treat the special case $\boldsymbol{f}=\mathbf{0}$ in $L^{p^{\prime}}\left(I,\left(V \cap \cap_{j} H\right)^{*}\right)$.

1. Galerkin approximation: Based on the separability of $V \cap_{j} H$ (cf. Proposition 2.9 (i)) there exists a sequence $\left(v_{i}\right)_{i \in \mathbb{N}} \subseteq V \cap_{j} H$ which is dense in $V \cap_{j} H$. Due to the density of $R(j)$ in $H$ and the Gram-Schmidt process we can additionally assume that $\left(j v_{i}\right)_{i \in \mathbb{N}} \subseteq H$ is dense and orthonormal in $H$. We set $V_{n}:=\operatorname{span}\left\{v_{1}, \ldots, v_{n}\right\}$ equipped with $\|\cdot\|_{V}$ and $H_{n}:=j\left(V_{n}\right)$ equipped with $(\cdot, \cdot)_{H}$. Denote by $j_{n}: V_{n} \rightarrow H_{n}$ the restriction of $j$ to $V_{n}$ and by $R_{n}: H_{n} \rightarrow H_{n}^{*}$ the corresponding Riesz isomorphism with respect to $(\cdot, \cdot)_{H}$. As $j_{n}$ is an isomorphism, the triple $\left(V_{n}, H_{n}, j_{n}\right)$ is an evolution triple with canonical embedding $e_{n}:=j_{n}^{*} R_{n} j_{n}: V_{n} \rightarrow V_{n}^{*}$. Moreover, we set

$$
\mathcal{X}_{n}:=L^{p}\left(I, V_{n}\right), \quad \mathcal{W}_{n}:=W_{e_{n}}^{1, p, p^{\prime}}\left(I, V_{n}, V_{n}^{*}\right), \quad \mathcal{Y}_{n}:=C^{0}\left(\bar{I}, H_{n}\right)
$$

Then, Proposition 2.23 provides the embedding $\boldsymbol{j}_{c}^{n}: \mathcal{W}_{n} \rightarrow \mathcal{Y}_{n}$ and the generalized integration by parts formula with respect to $\mathcal{W}_{n}$. 
We are seeking approximative solutions $\boldsymbol{y}_{n} \in \mathcal{W}_{n}$ which solve the Galerkin system

$$
\begin{aligned}
\int_{I}\left\langle\frac{d_{e_{n}} \boldsymbol{y}_{n}}{\mathrm{~d} t}(t), \boldsymbol{\varphi}(t)\right\rangle_{V \cap_{j} H} \mathrm{~d} t+\int_{I}\left\langle A(t)\left(\boldsymbol{y}_{n}(t)\right), \boldsymbol{\varphi}(t)\right\rangle_{V \cap_{j} H} \mathrm{~d} t=0 & \forall \boldsymbol{\varphi} \in \mathcal{X}_{n}, \\
\left(\boldsymbol{j}_{c}^{n} \boldsymbol{y}_{n}\right)(0)=\boldsymbol{y}_{0}^{n} & \text { in } H_{n},
\end{aligned}
$$

where $\boldsymbol{y}_{0}^{n}:=\sum_{i=1}^{n}\left(\boldsymbol{y}_{0}, j v_{i}\right)_{H} j v_{i}$.

\section{Existence of Galerkin solutions:}

It is straightforward to check that $\boldsymbol{y}_{n} \in \mathcal{W}_{n}$ iff

$$
\boldsymbol{y}_{n}=\sum_{i=1}^{n} \alpha_{i}^{n} v_{i} \text { with } \alpha_{i}^{n} \in W^{1, p^{\prime}}(I) \quad \text { and } \quad \frac{d_{e_{n}} \boldsymbol{y}_{n}}{\mathrm{~d} t}=\sum_{i=1}^{n} \frac{d \alpha_{i}^{n}}{\mathrm{~d} t} e_{n} v_{i} \text { in } L^{p^{\prime}}\left(I, V_{n}^{*}\right) \text {. }
$$

Thus, defining $\boldsymbol{f}^{n}: I \times \mathbb{R}^{n} \rightarrow \mathbb{R}^{n}$ by $\boldsymbol{f}^{n}(t, \boldsymbol{\alpha}):=\left(\left\langle A(t)\left(\sum_{k=1}^{n} \alpha_{k} v_{k}\right), v_{i}\right\rangle_{V \cap_{j} H}\right)_{i=1, \ldots, n}$ for almost every $t \in I$ and all $\alpha=\left(\alpha_{i}\right)_{i=1, \ldots, n} \in \mathbb{R}^{n}$, one sees, that (4.4) can be rewritten as a system of ordinary differential equations

$$
\begin{aligned}
\frac{d \boldsymbol{\alpha}^{n}}{\mathrm{~d} t}(s) & =\boldsymbol{f}^{n}\left(s, \boldsymbol{\alpha}^{n}(s)\right) & & \text { in } \mathbb{R}^{n} \text { for a.e. } s \in I, \\
\boldsymbol{\alpha}^{n}(0) & =\left(\left(\boldsymbol{y}_{0}, j v_{i}\right)\right)_{i=1, \ldots, n} & & \text { in } \mathbb{R}^{n} .
\end{aligned}
$$

From the assumptions (C.1) and (C.2) we deduce that the system (4.6) satisfies the standard Carathéodory conditions and by assumption (C.3) additionally a local majorant condition required in Carathéodory's existence theorem (cf. [9, Theorem 5.2]). The latter provides a maximal time horizon $T_{n} \in(0, T]$ and an absolutely continuous solution $\boldsymbol{\alpha}^{n}:\left[0, T_{n}\right) \rightarrow \mathbb{R}^{n}$ of (4.6) restricted to $\left[0, T_{n}\right)$. From (C.3) and $\boldsymbol{\alpha}^{n} \in C^{0}\left([0, t], \mathbb{R}^{n}\right)$ for all $0<t<T_{n}$ we infer that $\frac{d \boldsymbol{\alpha}^{n}}{\mathrm{~d} t}=\boldsymbol{f}^{n}\left(\cdot, \boldsymbol{\alpha}^{n}\right) \in L^{p^{\prime}}\left((0, t), \mathbb{R}^{n}\right)$ for all $0<t<T_{n}$. We set $\boldsymbol{y}_{n}:=\sum_{i=1}^{n} \alpha_{i}^{n} v_{i}$. Then, $\boldsymbol{y}_{n} \in W_{e_{n}}^{1, p, p^{\prime}}\left((0, t), V_{n}, V_{n}^{*}\right)$ for all $0<t<T_{n}$ (cf. (4.5)). Suppose $T_{n}<T$. We integrate the inner product of (4.6) and $\boldsymbol{\alpha}^{n}(s) \in \mathbb{R}^{n}$ with respect to $s \in[0, t]$, where $0<t \leq T_{n}$, apply the generalized integration by parts formula with respect to $W_{e_{n}}^{1, p, p^{\prime}}\left((0, t), V_{n}, V_{n}^{*}\right)$ (cf. Proposition 2.23), and use $\boldsymbol{j}_{c}^{n}=\boldsymbol{j}$ on $W_{e_{n}}^{1, p, p^{\prime}}\left((0, t), V_{n}, V_{n}^{*}\right)$, to obtain

$$
\frac{1}{2}\left\|\left(\boldsymbol{j} \boldsymbol{y}_{n}\right)(t)\right\|_{H}^{2}+\int_{0}^{t}\left\langle A(s)\left(\boldsymbol{y}_{n}(s)\right), \boldsymbol{y}_{n}(s)\right\rangle_{V \cap_{j} H} \mathrm{~d} s \leq \frac{1}{2}\left\|\boldsymbol{y}_{0}^{n}\right\|_{H}^{2} \leq \frac{1}{2}\left\|\boldsymbol{y}_{0}\right\|_{H}^{2}
$$

for all $t \in\left[0, T_{n}\right)$. By $\overline{\boldsymbol{y}}_{n}: \bar{I} \rightarrow V_{n}$ we denote the extension of $\boldsymbol{y}_{n}:\left[0, T_{n}\right) \rightarrow V_{n}$ by zero outside $\left[0, T_{n}\right)$. Thus, our extension satisfies

$$
\frac{1}{2}\left\|\left(j \bar{y}_{n}\right)(t)\right\|_{H}^{2}+\left\langle\mathcal{A} \bar{y}_{n}, \bar{y}_{n} \chi_{[0, t]}\right\rangle \mathcal{X} \leq \frac{1}{2}\left\|\boldsymbol{y}_{0}\right\|_{H}^{2}
$$


for all $t \in \bar{I}$. From (4.8) and the Bochner coercivity with respect to $\mathbf{0} \in \mathcal{X}^{*}$ and $\boldsymbol{y}_{0} \in H$ of $\mathcal{A}: D(\mathcal{A}) \subseteq \mathcal{X} \rightarrow \mathcal{X}^{*}$ with $D(\mathcal{A})=\mathcal{X} \cap j \mathcal{Y}$ we obtain an $n$ independent constant $M>0$ such that

$$
\left\|\boldsymbol{y}_{n}\right\|_{L^{p}\left(\left(0, T_{n}\right), V \cap_{j} H\right) \cap_{j} L^{\infty}\left(\left(0, T_{n}\right), H\right)}=\left\|\overline{\boldsymbol{y}}_{n}\right\| \boldsymbol{X}_{\cap_{j}} \mathcal{Y} \leq M
$$

In consequence, $\boldsymbol{\alpha}^{n} \in L^{\infty}\left(\left(0, T_{n}\right), \mathbb{R}^{n}\right)$ and therefore $\frac{d \boldsymbol{\alpha}^{n}}{\mathrm{~d} t}=\boldsymbol{f}^{n}\left(\cdot, \boldsymbol{\alpha}^{n}\right) \in L^{p^{\prime}}\left(\left(0, T_{n}\right), \mathbb{R}^{n}\right)$ due to (C.3). The fundamental theorem of calculus now yields $\alpha^{n} \in C^{0}\left(\left[0, T_{n}\right], \mathbb{R}^{n}\right)$. Hence, we can apply Caratheodory's theorem once more with initial value $\alpha^{n}\left(T_{n}\right) \in$ $\mathbb{R}^{n}$, to obtain an extension of $\boldsymbol{\alpha}^{n}$ to a solution of (4.6) on $\left[0, T_{n}+\varepsilon\right]$, with $\varepsilon>0$. This contradicts the maximality of $T_{n}>0$ and we conclude $T_{n}=T$. In particular, the estimates

$$
\left\|\boldsymbol{y}_{n}\right\| \boldsymbol{X}_{\cap_{j}} \mathcal{Y} \leq M \quad \text { and } \quad\left\|\mathcal{A}_{n}\right\|_{\boldsymbol{X}^{*}} \leq M^{\prime}
$$

hold true, where we used the boundedness of $\mathcal{A}: \mathcal{X} \cap_{j} \mathcal{Y} \rightarrow \mathcal{X}^{*}$ according to Lemma 3.13 (i) for the second estimate.

\section{Passage to the limit:}

3.1 Convergence of the Galerkin solutions: From the a-priori estimates (4.9) and Proposition 2.13 we obtain a not relabelled subsequence $\left(\boldsymbol{y}_{n}\right)_{n \in \mathbb{N}} \subseteq \mathcal{X} \cap \boldsymbol{j}$ as well as elements $\boldsymbol{y} \in \mathcal{X} \cap_{\boldsymbol{j}} \mathcal{Y}$ and $\boldsymbol{g} \in L^{p^{\prime}}\left(I,\left(V \cap_{j} H\right)^{*}\right)$ such that

$$
\begin{aligned}
\boldsymbol{y}_{n} \stackrel{n \rightarrow \infty}{\rightarrow} \boldsymbol{y} & \text { in } \mathcal{X}, \\
\boldsymbol{j} \boldsymbol{y}_{n} \stackrel{*}{\rightarrow} \boldsymbol{j} \boldsymbol{y} & \text { in } \mathcal{Y}, \quad(n \rightarrow \infty), \\
\mathcal{A} \boldsymbol{y}_{n} \stackrel{n \rightarrow \infty}{\rightarrow} R \boldsymbol{g} & \text { in } \mathcal{X}^{*} .
\end{aligned}
$$

3.2 Regularity and trace of the weak limit: Let $v \in V_{k}, k \in \mathbb{N}$, and $\varphi \in C^{\infty}(\bar{I})$ with $\varphi(T)=0$. Testing (4.4) for $n \geq k$ by $v \varphi \in \mathcal{X}_{k} \subseteq \mathcal{X}_{n}$, and a subsequent application of the generalized integration by parts formula with respect to $\mathcal{W}_{n}$ (cf. Proposition 2.23) yields

$$
\int_{I}\left\langle A(s)\left(\boldsymbol{y}_{n}(s)\right), v\right\rangle_{V \cap_{j} H} \varphi(s) \mathrm{d} s=\int_{I}\left(\left(\boldsymbol{j} \boldsymbol{y}_{n}\right)(s), j v\right)_{H} \varphi^{\prime}(s) \mathrm{d} s+\left(\boldsymbol{y}_{0}^{n}, j v\right)_{H} \varphi(0) .
$$

By passing with $n \geq k$ to infinity, using (4.10), Proposition 2.13 and $\boldsymbol{y}_{0}^{n} \stackrel{n \rightarrow \infty}{\rightarrow} \boldsymbol{y}_{0}$ in $H$, we obtain

$$
\int_{I}\langle\boldsymbol{g}(s), v\rangle_{V \cap_{j} H} \varphi(s) \mathrm{d} s=\int_{I}((\boldsymbol{j} \boldsymbol{y})(s), j v)_{H} \varphi^{\prime}(s) \mathrm{d} s+\left(\boldsymbol{y}_{0}, j v\right)_{H} \varphi(0)
$$

for all $v \in \bigcup_{k \in \mathbb{N}} V_{k}$ and $\varphi \in C^{\infty}(\bar{I})$ with $\varphi(T)=0$. Choosing $\varphi \in C_{0}^{\infty}(I)$ in (4.11), we have due to Definition 2.22 and Proposition 2.23

$\boldsymbol{y} \in \mathcal{W} \quad$ with $\quad \frac{d_{e} \boldsymbol{y}}{\mathrm{d} t}=-\boldsymbol{g} \quad$ in $L^{p^{\prime}}\left(I,\left(V \cap \cap_{j} H\right)^{*}\right) \quad$ and $\quad \boldsymbol{j}_{c} \boldsymbol{y} \in C^{0}(\bar{I}, H)$. 
Thus, we are allowed to apply the generalized integration by parts formula with respect to $\mathcal{W}$ in (4.11) in the case $\varphi \in C^{\infty}(\bar{I})$ with $\varphi(T)=0$ and $\varphi(0)=1$, which yields for all $v \in \bigcup_{k \in \mathbb{N}} V_{k}$

$$
\left(\left(\boldsymbol{j}_{c} \boldsymbol{y}\right)(0)-\boldsymbol{y}_{0}, j v\right)_{H}=0 .
$$

As $R(j)$ is dense in $H$ we deduce from (4.13) that

$$
\left(\boldsymbol{j}_{c} \boldsymbol{y}\right)(0)=\boldsymbol{y}_{0} \quad \text { in } H .
$$

\subsection{Pointwise weak convergence in $H$ :}

Now we show that $\left(\boldsymbol{j} \boldsymbol{y}_{n}\right)(t) \stackrel{n \rightarrow \infty}{\longrightarrow}(\boldsymbol{j} \boldsymbol{y})(t)$ in $H$ for almost every $t \in I$, which is the crucial new condition of Bochner pseudo-monotonicity compared to standard pseudo-monotonicity, apart from the boundedness in $\mathcal{Y}$. To this end, let us fix an arbitrary $t \in(0, T]$. From the a-priori estimate $\left\|\left(\boldsymbol{j} \boldsymbol{y}_{n}\right)(t)\right\|_{H} \leq M$ for all $t \in \bar{I}$ and $n \in \mathbb{N}$ (cf. (4.9)) we obtain the existence of a subsequence $\left(\left(\boldsymbol{j} \boldsymbol{y}_{n}\right)(t)\right)_{n \in \Lambda_{t}} \subseteq H$ with $\Lambda_{t} \subseteq \mathbb{N}$, initially depending on this fixed $t$, and an element $\boldsymbol{y}_{\Lambda_{t}} \in H$ such that

$$
\left(j y_{n}\right)(t) \stackrel{n \rightarrow \infty}{\rightarrow} y_{\Lambda_{t}} \text { in } H\left(n \in \Lambda_{t}\right) .
$$

For $v \in V_{k}, k \in \Lambda_{t}$, and $\varphi \in C^{\infty}(\bar{I})$ with $\varphi(0)=0$ and $\varphi(t)=1$, we test (4.4) for $n \geq k\left(n \in \Lambda_{t}\right)$ by $v \varphi \chi_{[0, t]} \in \mathcal{X}_{k} \subseteq \mathcal{X}_{n}$, integrate with respect to $s \in[0, t]$, use the generalized integration by parts formula in $\mathcal{W}_{n}$, and (2.11), to obtain for all $n \geq k$ with $n \in \Lambda_{t}$

$$
\int_{0}^{t}\left\langle A(s)\left(\boldsymbol{y}_{n}(s)\right), v\right\rangle_{V \cap_{j} H} \varphi(s) \mathrm{d} s=\int_{0}^{t}\left(\left(\boldsymbol{j} \boldsymbol{y}_{n}\right)(s), j v\right)_{H} \varphi^{\prime}(s) \mathrm{d} s-\left(\left(\boldsymbol{j} \boldsymbol{y}_{n}\right)(t), j v\right)_{H} .
$$

By passing for $n \geq k$ with $n \in \Lambda_{t}$ to infinity, using (4.10) and (4.15), we obtain

$$
\int_{0}^{t}\langle\boldsymbol{g}(s), v\rangle_{V \cap_{j} H} \varphi(s) \mathrm{d} s=\int_{0}^{t}((\boldsymbol{j} \boldsymbol{y})(s), j v)_{H} \varphi^{\prime}(s) \mathrm{d} s-\left(\boldsymbol{y}_{\Lambda_{t}}, j v\right)_{H}
$$

for all $v \in \bigcup_{k \in \Lambda_{t}} V_{k}$. From (4.12) and the generalized integration by parts formula in $\mathcal{W}$ we also obtain

$$
\left(\left(\boldsymbol{j}_{c} \boldsymbol{y}\right)(t)-\boldsymbol{y}_{\Lambda_{t}}, j v\right)_{H}=0
$$

for all $v \in \bigcup_{k \in \Lambda_{t}} V_{k}$. Thanks to $V_{k} \subseteq V_{k+1}$ for all $k \in \mathbb{N}$ we get $\bigcup_{k \in \Lambda_{t}} V_{k}=$ $\bigcup_{k \in \mathbb{N}} V_{k}$. Thus, $j\left(\bigcup_{k \in \Lambda_{t}} V_{k}\right)$ is dense in $H$ and (4.16) yields that $(\boldsymbol{j} \boldsymbol{y})(t)=\boldsymbol{y}_{\Lambda_{t}}$ in $H$. Consequently, we deduce from (4.15) that

$$
\left(\boldsymbol{j} \boldsymbol{y}_{n}\right)(t) \stackrel{n \rightarrow \infty}{\rightarrow}\left(\boldsymbol{j}_{c} \boldsymbol{y}\right)(t) \quad \text { in } H\left(n \in \Lambda_{t}\right) .
$$

As this argumentation stays valid for each weakly convergent subsequence of $\left(\left(\boldsymbol{j} \boldsymbol{y}_{n}\right)(t)\right)_{n \in \mathbb{N}} \subseteq H,\left(\boldsymbol{j}_{c} \boldsymbol{y}\right)(t) \in H$ is weak accumulation point of each weakly converging subsequence of $\left(\left(\boldsymbol{j} \boldsymbol{y}_{n}\right)(t)\right)_{n \in \mathbb{N}} \subseteq H$. The standard convergence principle 
(cf. [8, Kap. I, Lemma 5.4]) yields $\Lambda_{t}=\mathbb{N}$ in (4.17). Since $\left(\boldsymbol{j}_{c} \boldsymbol{y}\right)(t)=(\boldsymbol{j} \boldsymbol{y})(t)$ in $H$ for almost every $t \in I$, we conclude for almost every $t \in I$

$$
\left(\boldsymbol{j} \boldsymbol{y}_{n}\right)(t) \stackrel{n \rightarrow \infty}{\rightarrow}(\boldsymbol{j} \boldsymbol{y})(t) \text { in } H
$$

3.4 Identification of $\mathcal{A y}$ and $R \boldsymbol{g}$ : Due to (4.7) in the case $t=T$ we have for all $n \in \mathbb{N}$

$$
\left\langle\mathcal{A} \boldsymbol{y}_{n}, \boldsymbol{y}_{n}\right\rangle \mathcal{X} \leq-\frac{1}{2}\left\|\left(\boldsymbol{j} \boldsymbol{y}_{n}\right)(T)\right\|_{H}^{2}+\frac{1}{2}\left\|\boldsymbol{y}_{0}\right\|_{H}^{2} .
$$

This inequality together with $(4.10)_{3},(4.14)$, (4.17) with $\Lambda_{t}=\mathbb{N}$ in the case $t=T$, the weak lower semi-continuity of $\|\cdot\|_{H}$, the generalized integration by parts formula in $\mathcal{W}$ and (4.12) yields

$$
\begin{aligned}
\limsup _{n \rightarrow \infty}\left\langle\mathcal{A} \boldsymbol{y}_{n}, \boldsymbol{y}_{n}-\boldsymbol{y}\right\rangle \boldsymbol{\mathcal { X }} & \leq-\frac{1}{2}\left\|\left(\boldsymbol{j}_{c} \boldsymbol{y}\right)(T)\right\|_{H}^{2}+\frac{1}{2}\left\|\left(\boldsymbol{j}_{c} \boldsymbol{y}\right)(0)\right\|_{H}^{2}-\int_{I}\langle\boldsymbol{g}(s), \boldsymbol{y}(s)\rangle_{V \cap_{j} H} \mathrm{~d} s \\
& =-\int_{I}\left\langle\frac{d_{e} \boldsymbol{y}}{\mathrm{d} t}(s)+\boldsymbol{g}(s), \boldsymbol{y}(s)\right\rangle_{V \cap_{j} H} \mathrm{~d} s=0 .
\end{aligned}
$$

As a result of (4.10), (4.18), (4.19) and the Bochner pseudo-monotonicity of $\mathcal{A}$ : $D(\mathcal{A}) \subseteq \mathcal{X} \rightarrow \mathcal{X}^{*}$ with $D(\mathcal{A})=\mathcal{X} \cap j \mathcal{Y}$, Lemma 3.7 (i) finally provides $\mathcal{A} \boldsymbol{y}=$ $R \boldsymbol{g}$ in $\mathcal{X}^{*}$. This completes the proof of Theorem 4.1.

\section{Examples}

In this section, we give two prototypical examples to which Theorem 4.1 and the notions developed in Sect. 3 can be applied. We emphasize that the existence results given in these two examples are not new, see e.g., $[1,14]$. The following examples shall merely illustrate that the conditions (C.1)-(C.5) are easily verifiable and quite general, and in what way the scope of application is extended by the treatment of pre-evolution triples.

Example 5.1. (Unsteady p-Navier-Stokes equation for $p \geq \frac{11}{5}$ ) Let $\Omega \subseteq \mathbb{R}^{3}$ be a bounded domain, $I:=(0, T)$, with $0<T<\infty$. Moreover, let $\mathcal{V}=\left\{\mathbf{v} \in C_{0}^{\infty}(\Omega)^{3} \mid\right.$ $\operatorname{div} \mathbf{v}=0\}, V$ the closure of $\mathcal{V}$ with respect to the gradient norm $\|\nabla \cdot\|_{L^{p}(\Omega)^{3 \times 3}}, H$ the closure of $\mathcal{V}$ with respect to $\|\cdot\|_{L^{2}(\Omega)^{3}}$ and let $S, B: V \rightarrow V^{*}$ be defined as in the introduction. Then, $\left(V, H, \mathrm{id}_{V}\right)$ is an evolution triple, $A:=S+B: V \rightarrow V^{*}$ satisfies (C.1)-(C.5) with $\mathscr{C} \equiv 0$ in (C.5) and its induced operator $\mathcal{A}: L^{p}(I, V) \cap L^{\infty}(I, H) \rightarrow$ $\left(L^{p}(I, V)\right)^{*}$ is bounded, Bochner pseudo-monotone and coercive. In addition, for arbitrary $\mathbf{u}_{0} \in H$ and $\boldsymbol{f} \in L^{p^{\prime}}\left(I, V^{*}\right)$ there exists a solution $\boldsymbol{u} \in W_{e}^{1, p, p^{\prime}}\left(I, V, V^{*}\right)$, where $e:=\left(\operatorname{id}_{V}\right)^{*} R_{H} \mathrm{id}_{V}$, of 


$$
\begin{aligned}
& \int_{I}\left\langle\frac{d_{e} \boldsymbol{u}}{\mathrm{d} t}(s), v\right\rangle_{V} \varphi(s) \mathrm{d} s+\int_{I} \int_{\Omega}(\mathbf{S}(\mathbf{D} \boldsymbol{u}(s))-\boldsymbol{u}(s) \otimes \boldsymbol{u}(s)): \mathbf{D v} \varphi(s) \mathrm{d} x \mathrm{~d} s \\
& \quad=\int_{I}\langle\boldsymbol{f}(s), \mathbf{v}\rangle_{V} \varphi(s) \mathrm{d} s
\end{aligned}
$$

for all $\mathbf{v} \in V$ and $\varphi \in C_{0}^{\infty}(I)$ with $\boldsymbol{u}(0)=\mathbf{u}_{0}$ in $H$.

Proof. Clearly, $\left(V, H\right.$, id) forms an evolution triple and $A: V \rightarrow V^{*}$ is bounded, pseudo-monotone and demi-continuous, see e.g., [1, Section 6], and thus satisfies (C.1), (C.2) and (C.4). (C.5) with $\mathscr{C} \equiv 0$ immediately follows from $\langle S \mathbf{v}, \mathbf{v}\rangle_{V}=\|\mathbf{v}\|_{V}^{p}$ and $\langle B \mathbf{v}, \mathbf{v}\rangle_{V}=0$ for every $\mathbf{v} \in V$. For (C.3) we first note that $\|S \mathbf{v}\|_{V^{*}} \leq\|\mathbf{v}\|_{V}^{p-1}$ and $\|B \mathbf{v}\|_{V^{*}} \leq\|\mathbf{v}\|_{L^{2 p^{\prime}}(\Omega)}^{2}$ for every $\mathbf{v} \in V$.

If $p \geq 3$, then $p-1 \geq 2$ and $\|\mathbf{v}\|_{L^{2 p^{\prime}}(\Omega)} \leq c\|\mathbf{v}\|_{V}$ for all $\mathbf{v} \in V$. Thus, using $a^{2} \leq(1+a)^{p-1} \leq 2^{p-2}\left(1+a^{p-1}\right)$ for all $a \geq 0$, we obtain $\|B \mathbf{v}\|_{V^{*}} \leq c\left(1+\|\mathbf{v}\|_{V}^{p-1}\right)$ for every $v \in V$.

If $p \in\left[\frac{11}{5}, 3\right)$, then by interpolation with $\frac{1}{\rho}=\frac{1-\theta}{p^{*}}+\frac{\theta}{2}$, where $\rho=p \frac{5}{3}, \theta=\frac{2}{5}$ and $p^{*}=\frac{3 p}{3-p}$, and using $a^{6 / 5} \leq(1+a)^{p-1} \leq 2^{p-2}\left(1+a^{p-1}\right)$ for all $a \geq 0$, as $\frac{6}{5} \leq p-1$, we obtain for all $v \in V$

$$
\|\mathbf{v}\|_{L^{\rho}(\Omega)^{3}}^{2} \leq\|\mathbf{v}\|_{H}^{\frac{4}{5}}\|\mathbf{v}\|_{L^{p^{*}}(\Omega)}^{\frac{6}{5}} \leq\|\mathbf{v}\|_{H}^{\frac{4}{5}}\left(1+\|\mathbf{v}\|_{L^{p^{*}}(\Omega)}\right)^{p-1} \leq c\|\mathbf{v}\|_{H}^{\frac{4}{5}}\left(1+\|\mathbf{v}\|_{V}^{p-1}\right) .
$$

Since also $\rho \geq 2 p^{\prime}$, we infer $\|B \mathbf{v}\|_{V^{*}} \leq c\|\mathbf{v}\|_{H}^{\frac{4}{5}}\left(1+\|\mathbf{v}\|_{V}^{p-1}\right)$ for every $v \in V$. Altogether, $A: V \rightarrow V^{*}$ satisfies (C.3) and meets the framework of Proposition 3.13 and Corollary 4.2, which in turn yield the assertion.

Example 5.2. (Unsteady p-Laplace equation with compact perturbation for $p \in$ $(1, \infty))$ Let $\Omega \subseteq \mathbb{R}^{d}$ be a bounded domain, $I:=(0, T)$, with $0<T<\infty$, and let $b: I \times \Omega \times \mathbb{R} \rightarrow \mathbb{R}$ be a function with the following properties:

(B.1) $b$ is measurable in its first two variables and continuous in its third variable.

(B.2) For some nonnegative functions $C_{1} \in L^{p^{\prime}}\left(I, L^{q}(\Omega)\right), q=\min \left\{2,\left(p^{*}\right)^{\prime}\right\}$, $C_{2} \in L^{p^{\prime}}\left(I, L^{\infty}(\Omega)\right)$ and $1 \leq r<\max \left\{2, p \frac{d+2}{d}\right\}$ holds

$$
|b(t, x, s)| \leq C_{1}(t, x)+C_{2}(t, x)(1+|s|)^{r-1}
$$

for almost every $(t, x) \in I \times \Omega$ and all $s \in \mathbb{R}$.

(B.3) For some functions $c_{1} \in L^{1}\left(I, L^{\infty}(\Omega)\right)$ and $c_{2} \in L^{1}\left(I \times \Omega, \mathbb{R}_{\geq 0}\right)$ holds

$$
b(t, x, s) \cdot s \geq c_{1}(t, x)|s|^{2}-c_{2}(t, x)
$$

for almost every $(t, x) \in I \times \Omega$ and all $s \in \mathbb{R}$.

Moreover, set $V=W_{0}^{1, p}(\Omega), H=L^{2}(\Omega)$ and let $A_{0}, B(t): V \cap H \rightarrow(V \cap H)^{*}$ be given via

$$
\left\langle A_{0} v, w\right\rangle_{V \cap H}:=\int_{\Omega}|\nabla v|^{p-2} \nabla v \cdot \nabla w \mathrm{~d} x
$$




$$
\langle B(t) v, w\rangle_{V \cap H}:=\int_{\Omega} b(t, x, v) \cdot w \mathrm{~d} x
$$

for almost every $t \in I$ and all $v, w \in V \cap H$. Then, $\left(V, H, \mathrm{id}_{V \cap H}\right)$ forms a preevolution triple, $A(t):=A_{0}+B(t): V \cap H \rightarrow(V \cap H)^{*}, t \in I$, satisfies (C.1)-(C.5) with $\mathscr{C}(s)=s^{2}$ in (C.5) and its induced operator $\mathcal{A}: L^{p}(I, V \cap H) \cap L^{\infty}(I, H) \rightarrow$ $\left(L^{p}(I, V \cap H)\right)^{*}$ is bounded, Bochner pseudo-monotone and Bochner coercive. In addition, for arbitrary $\mathbf{u}_{0} \in L^{2}(\Omega)$ and $f \in L^{p^{\prime}}\left(I,(V \cap H)^{*}\right)$ there exists a solution $\boldsymbol{u} \in W_{e}^{1, p, p^{\prime}}\left(I, V \cap H,(V \cap H)^{*}\right)$, where $e:=\left(\operatorname{id}_{V \cap H}\right)^{*} R_{H} \operatorname{id}_{V \cap H}$, of

$$
\begin{aligned}
& \int_{I}\left\langle\frac{d_{e} \boldsymbol{u}}{\mathrm{d} t}(s), v\right\rangle_{V \cap H} \varphi(s) \mathrm{d} s+\int_{I} \int_{\Omega}\left[|\nabla \boldsymbol{u}(s)|^{p-2} \nabla \boldsymbol{u}(s) \cdot \nabla v+b(s, x, \boldsymbol{u}(s)) \cdot v\right] \varphi(s) \mathrm{d} x \mathrm{~d} s \\
& =\int_{I}\langle\boldsymbol{f}(s), v\rangle_{V \cap H} \varphi(s) \mathrm{d} s
\end{aligned}
$$

for all $v \in V \cap H$ and $\varphi \in C_{0}^{\infty}(I)$ with $\mathbf{u}(0)=\mathbf{u}_{0}$ in $H$. As $C_{0}^{\infty}(\Omega)$ is dense in $V \cap H$ we infer from (5.3) the distributional identity

$$
\partial_{t} \boldsymbol{u}-\operatorname{div}\left(|\nabla \boldsymbol{u}|^{p-2} \nabla \boldsymbol{u}\right)+b(\cdot, \cdot, \boldsymbol{u})=\boldsymbol{f} \quad \text { in } \mathcal{D}^{\prime}(I \times \Omega) .
$$

Proof. Clearly, $(V, H$, id $)=\left(W_{0}^{1, p}(\Omega), L^{2}(\Omega), L^{1}(\Omega)\right.$, id, id $)$ forms a pre-evolution triple. As $A_{0}: V \cap H \rightarrow(V \cap H)^{*}$ already meets the framework of Corollary 4.2 (cf. [19, Lemmata 1.26 and 1.28]), it remains to ensure that $B(t): V \cap H \rightarrow(V \cap H)^{*}$ satisfies (C.1)-(C.4) and $A(t): V \cap H \rightarrow(V \cap H)^{*}$ the semi-coercivity condition (C.5) with $\mathscr{C}(s)=s^{2}$. We restrict ourselves to the case $p \in\left(1, \frac{2 d}{d+2}\right]$, since the case $p>\frac{2 d}{d+2}$ is already treated in [1, Theorem 6.2] or [18, Proposition 8.37] with the help of the evolution triple $(V, H, i d)$ and requires only obvious modifications to adjust to our framework. From (B.1) and (B.2) in conjunction with the theory of Nemyckii operators (cf. [18, Theorem 1.43]) we deduce for almost every $t \in I$ the welldefinedness and continuity of $F_{t}: L^{\rho}(\Omega) \rightarrow L^{2}(\Omega)$, where $\rho:=\max \{1,2(r-1)\}$, given via $\left(F_{t} v\right)(x):=b(t, x, v(x))$ for almost every $x \in \Omega$ and all $v \in L^{\rho}(\Omega)$. In fact, using (B.2) and $(1+a)^{2(r-1)} \leq(1+a)^{\rho}$ for all $a \geq 0$, we obtain

$$
\begin{aligned}
\left\|F_{t} v\right\|_{L^{2}(\Omega)} & \leq\left\|C_{1}(t, \cdot)\right\|_{L^{2}(\Omega)}+\left\|C_{2}(t, \cdot)\right\|_{L^{\infty}(\Omega)}\left(\int_{\Omega}(1+|v|)^{2(r-1)} \mathrm{d} x\right)^{\frac{1}{2}} \\
& \leq\left\|C_{1}(t, \cdot)\right\|_{L^{2}(\Omega)}+\left\|C_{2}(t, \cdot)\right\|_{L^{\infty}(\Omega)}\left(C(\Omega)+\|v\|_{L^{\rho}(\Omega)}^{\frac{\rho}{2}}\right)
\end{aligned}
$$

for almost every $t \in I$ and all $v \in V \cap H$. Due to $\rho<2, V \hookrightarrow \hookrightarrow L^{1}(\Omega)$ and Vitali's theorem we get $V \cap H \hookrightarrow \hookrightarrow L^{\rho}(\Omega)$, i.e., $\operatorname{id}_{V \cap H}: V \cap H \rightarrow L^{\rho}(\Omega)$ is strongly continuous. From the latter, $B(t)=\left(\operatorname{id}_{V \cap H}\right)^{*} F_{t}\left(\operatorname{id}_{V \cap H}\right)$ and the continuity of both $\left(\operatorname{id}_{V \cap H}\right)^{*}: H \rightarrow(V \cap H)^{*}$ and $F_{t}: L^{\rho}(\Omega) \rightarrow L^{2}(\Omega)$ we infer that $B(t)$ : $V \cap H \rightarrow(V \cap H)^{*}$ is strongly continuous and thus pseudo-monotone. Thus, we 
verified (C.1), (C.4) and (C.3) with $\mathscr{B}(s):=s^{\frac{\rho}{2}}, \alpha(t):=\left\|C_{2}(t, \cdot)\right\|_{L^{\infty}(\Omega)}, \beta(t):=0$ and $\gamma(t):=\left\|C_{1}(t, \cdot)\right\|_{L^{2}(\Omega)}+C(\Omega)\left\|C_{2}(t, \cdot)\right\|_{L^{\infty}(\Omega)}$ (cf. (5.4)). Condition (C.2) is a consequence of Fubini's theorem. Using (B.3), the semi-coercivity condition (C.5) follows by

$\langle A(t) v, v\rangle_{V}=\left\langle A_{0} v, v\right\rangle_{V}+\langle B(t) v, v\rangle_{V} \geq\|v\|_{V}^{p}-\left\|c_{1}(t, \cdot)\right\|_{L^{\infty}(\Omega)}\|v\|_{H}^{2}-\left\|c_{2}(t, \cdot)\right\|_{L^{1}(\Omega)}$

for almost all $t \in I$ and all $v \in V \cap H$. Altogether, $A(t):=A_{0}+B(t): V \cap H \rightarrow$ $(V \cap H)^{*}, t \in I$, meets the framework of Proposition 3.13 and Corollary 4.2, which yield the assertion.

\section{Acknowledgements}

Open Access funding provided by Projekt DEAL. We would like to thank the referee for the helpful comments which improved the presentation of the paper.

Open Access. This article is licensed under a Creative Commons Attribution 4.0 International License, which permits use, sharing, adaptation, distribution and reproduction in any medium or format, as long as you give appropriate credit to the original author(s) and the source, provide a link to the Creative Commons licence, and indicate if changes were made. The images or other third party material in this article are included in the article's Creative Commons licence, unless indicated otherwise in a credit line to the material. If material is not included in the article's Creative Commons licence and your intended use is not permitted by statutory regulation or exceeds the permitted use, you will need to obtain permission directly from the copyright holder. To view a copy of this licence, visit http://creativecommons.org/licenses/ by/4.0/.

Publisher's Note Springer Nature remains neutral with regard to jurisdictional claims in published maps and institutional affiliations.

\section{REFERENCES}

[1] E. BÄUMLE AND M. RŮð̆CKA, Note on the existence theory for evolution equations with pseudomonotone operators, Ric. Mat. 66 (2017), no. 1, 35-50.

[2] C. BennetT AND R. Sharpley, Interpolation of operators, Pure and Applied Mathematics, vol. 129, Academic Press, Inc., Boston, MA, 1988.

[3] F. BOYER AND P. FABRIE, Mathematical tools for the study of the incompressible Navier-Stokes equations and related models, Applied Mathematical Sciences, vol. 183, Springer, New York, 2013.

[4] H. BREZIS, Équations et inéquations non linéaires dans les espaces vectoriels en dualité, Ann. Inst. Fourier (Grenoble) 18 (1968), no. fasc. 1, 115-175.

[5] F. E. BROWDER, Nonlinear elliptic boundary value problems, Bull. Am. Math. Soc. 69 (1963), $862-874$.

[6] F. E. BROWDER, Nonlinear maximal monotone operators in Banach space, Math. Ann. 175 (1968), 89-113.

[7] J. Diestel AND J. J. UHL, JR., Vector measures, American Mathematical Society, Providence, R.I., 1977, With a foreword by B. J. Pettis, Mathematical Surveys, No. 15.

[8] H. GAJEWSKI, K. GRÖGER, AND K. ZACHARIAS, Nichtlineare Operatorgleichungen und Operatordifferentialgleichungen, Akademie-Verlag, Berlin, 1974.

[9] J. K. HALE, Ordinary differential equations, Second ed., Robert E. Krieger Publishing Co., Inc., Huntington, NY, 1980. 
[10] N. HIRANO, Nonlinear Volterra equations with positive kernels, Nonlinear and convex analysis (Santa Barbara, Calif., 1985), Lecture Notes in Pure and Appl. Math., vol. 107, Dekker, New York, 1987, pp. 83-98.

[11] N. HIRANO, Nonlinear evolution equations with nonmonotonic perturbations, Nonlinear Anal. 13 (1989), no. 6, 599-609.

[12] A. KALTENBACH, Verallgemeinerte nichtlineare Evolutionsgleichungen, Master's thesis, Institute of Applied Mathematics, Albert-Ludwigs-University Freiburg, 2019.

[13] R. LANDES AND V. MUSTONEN, A strongly nonlinear parabolic initial-boundary value problem, Ark. Mat. 25 (1987), no. 1, 29-40.

[14] J.- L. LiONS, Quelques méthodes de résolution des problèmes aux limites non linéaires, Dunod; Gauthier-Villars, Paris, 1969.

[15] G. J. MINTY, On a "monotonicity" method for the solution of non-linear equations in Banach spaces, Proc. Natl. Acad. Sci. USA 50 (1963), 1038-1041.

[16] N. S. PAPAGEORGIOU, On the existence of solutions for nonlinear parabolic problems with nonmonotone discontinuities, J. Math. Anal. Appl. 205 (1997), no. 2, 434-453.

[17] J. M. RAKOTOSON AND R. TEMAM, An optimal compactness theorem and application to ellipticparabolic systems, Appl. Math. Lett. 14 (2001), no. 3, 303-306.

[18] T. RoubíceK, Nonlinear partial differential equations with applications, International Series of Numerical Mathematics, vol. 153, Birkhäuser Verlag, Basel, 2005.

[19] M. RŮŽCKA, Nonlinear functional analysis. An introduction. (Nichtlineare Funktionalanalysis. Eine Einführung.), Berlin: Springer. xii, 208 p., 2004 (German).

[20] N. SHIOJI, Existence of periodic solutions for nonlinear evolution equations with pseudomonotone operators, Proc. Amer. Math. Soc. 125 (1997), no. 10, 2921-2929.

[21] R. E. SHOWALTER, Monotone operators in Banach space and nonlinear partial differential equations, Mathematical Surveys and Monographs, vol. 49, American Mathematical Society, Providence, RI, 1997.

[22] K. YosidA, Functional analysis, Sixth ed., Grundlehren der Mathematischen Wissenschaften [Fundamental Principles of Mathematical Sciences], vol. 123, Springer-Verlag, Berlin-New York, 1980.

[23] E. ZEIDLER, Nonlinear functional analysis and its applications. II/A Linear monotone operators., Springer, New York 1990.

[24] E. ZEIDLER, Nonlinear functional analysis and its applications. II/B Nonlinear monotone operators., Springer, New York 1990.

\author{
A. Kaltenbach and M. Ruzička \\ Institute of Applied Mathematics \\ Albert-Ludwigs-University Freiburg \\ Ernst-Zermelo-Straße 1 \\ 79104 Freiburg \\ Germany \\ E-mail: rose@mathematik.uni-freiburg.de
}

A. Kaltenbach

E-mail: alex.kaltenbach@mathematik.uni-freiburg.de 